\title{
THE
}

UNIVERSITY

University of Rhode Island

OF RHODE ISLAND

DigitalCommons@URI

3-29-2002

\section{Surface Roughness and Size Effects in Quantized Films}

\author{
A. E. Meyerovich \\ University of Rhode Island, sfo101@uri.edu
}

I. V. Ponomarev

Follow this and additional works at: https://digitalcommons.uri.edu/phys_facpubs

Terms of Use

All rights reserved under copyright.

\section{Citation/Publisher Attribution}

Meyerovich, A. E., \& Ponomarev, I. V. (2002). Surface Roughness and Size Effects in Quantized Films.

Phys. Rev. B, 65(15), 155413. doi: 10.1103/PhysRevB.65.155413

Available at: http://dx.doi.org/10.1103/PhysRevB.65.155413

This Article is brought to you for free and open access by the Physics at DigitalCommons@URI. It has been accepted for inclusion in Physics Faculty Publications by an authorized administrator of DigitalCommons@URI. For more information, please contact digitalcommons-group@uri.edu. 


\title{
Surface roughness and size effects in quantized films
}

\author{
A. E. Meyerovich and I. V. Ponomarev \\ Department of Physics, University of Rhode Island, 2 Lippitt Rd., Kingston, Rhode Island 02881-0817
}

(Received 2 October 2001; published 29 March 2002)

\begin{abstract}
The effect of random surface roughness on quantum size effects in thin films is discussed. The conductivity of quantized metal films is analyzed for different types of experimentally identified correlation functions of surface inhomogeneities including the Gaussian, exponential, power-law correlators, and correlators with a power-law decay of the power density spectral function. The dependence of the conductivity $\sigma$ on the film thickness $L$, correlation radius of inhomogeneities $R$, and the fermion density is investigated. The goal is to help in extracting surface parameters from transport measurements and to determine the importance of the choice of the proper surface correlator for transport theory. A peculiar size effect is predicted for quantized films with large correlation radius of random surface corrugation. The effect exists for inhomogeneities with Gaussian and exponential power spectrum; if the decay of power spectrum is slow, the films exhibit usual quantum size effect. The conductivity $\sigma$ exhibits well-pronounced oscillations as a function of channel width $L$ or density of fermions, and large steps as a function of the correlation radius $R$. These oscillations and steps are explained and their positions identified. This phenomenon, which is reminiscent of magnetic breakthrough, can allow direct observation of the quantum size effect in conductivity of nanoscale metal films. The only region with a nearly universal behavior of transport is the region in which particle wavelength is close to the correlation radius of surface inhomogeneities.
\end{abstract}

DOI: 10.1103/PhysRevB.65.155413

PACS number(s): 72.10.Fk, 73.23.Ad, 73.50.Bk

\section{INTRODUCTION}

Progress in material technology, especially in nanofabrication, ultrathin-film manufacturing, ultraclean and highvacuum systems, etc., requires better understanding of boundary scattering in physical processes. The boundary effects should be an integral part of any study of quantum wires, wells, and films. Boundary scattering is especially important for transport in ultrathin and/or clean systems in which the particle mean free path is comparable to the system size.

Below we consider the effect of random surface roughness on quantum transport in quantized quasi-twodimensional (quasi-2D) systems such as, for example, ultrathin metal films. The main issue is to find how sensitive is the transport along such film to the statistical properties of random surface inhomogeneities (thickness fluctuations). An important by-product of our systematic comparison of different classes of random surface inhomogeneities is the prediction of a new type of size effect in quantized films. This effect manifests itself as large oscillations of conductivity $\sigma$ as a function of the film thickness $L$. In contrast to the usual quantum size effect (QSE), the peaks can be observed only at relatively large values of $L$. The distance between the peaks is large and is roughly proportional to $L^{2}$. The observation of this QSE opens an experimental method of identification of the type of surface roughness.

The choice of quasi-2D systems is explained by a desire to avoid divergence of surface fluctuations and strong localization effects which are inherent to 1D systems and make a systematic quantitative study of the effect of surface inhomogeneities on transport virtually impossible. In contrast to 1D systems, the randomly fluctuating 2D surfaces are practically stable while the localization length in systems with weak surface roughness is exponentially large. (In general, the transport problems are more interesting in systems with weak rather than with strong roughness. Transport in systems with strong roughness is trivial: each wall collision completely dephases the particles and the mean free path cannot exceed the distance between the walls.)

The prevalent way to characterize the random surface roughness and/or thickness fluctuations is to use the correlation function of surface inhomogeneities:

$$
\zeta(\mathbf{s}) \equiv \zeta(|\mathbf{s}|)=\left\langle\xi\left(\mathbf{s}_{1}\right) \xi\left(\mathbf{s}_{1}+\mathbf{s}\right)\right\rangle \equiv A^{-1} \int \xi\left(\mathbf{s}_{1}\right) \xi\left(\mathbf{s}_{1}+\mathbf{s}\right) d \mathbf{s}_{1},
$$

where the vector $\mathbf{s}$ gives the $2 \mathrm{D}$ coordinates along the surface, $\xi(\mathbf{s})$ describes the deviation of the position of the surface in the point with $2 \mathrm{D}$ coordinates $\mathbf{s}$ from its average position, $\langle\xi(\mathbf{s})\rangle=0$, and $A$ is the averaging area. Here it is assumed that the correlation properties of the surface do not depend on direction. Two main characteristics of the surface correlation functions $\zeta$ are the average amplitude ("height") $l$ and correlation radius ("size") $R$ of surface inhomogeneities.

Any transport theory for systems with rough boundaries should provide the explicit dependence of the particle mean free path (or the conductivity along the walls) on the correlator of surface inhomogeneities $\zeta(s)$. Without bulk scattering, the conductivity $\sigma$ is determined by the relation between three length scales: particle wavelength, $\Lambda$; width of the channel, $L$; and correlation radius of inhomogeneities, $R$. If the roughness is weak, the fourth length parameter $l$ enters the conductivity as a coefficient:

$$
\sigma=\frac{2 e^{2}}{\hbar} \frac{L^{2}}{l^{2}} f(\Lambda, L, R)
$$


Note that this $2 \mathrm{D}$ conductivity differs by a length unit from the usual 3D conductivity and, as a result, has a dimensionality of conductance.

The form of the surface correlator $\zeta(s)$ can vary from surface to surface. Most of the theoretical calculations assume that this correlator is Gaussian. The numerical simulations, on the other hand, often rely on various generators for random rough surfaces without paying much attention to the correlation function of the generated inhomogeneities. Both approaches are not satisfactory since the experiments on surface scattering and diffraction patterns show that real surfaces exhibit surface correlators (1) of various forms. ${ }^{1,2}$ Even one and the same film can exhibit various correlation properties on different stages of growth. As a result, the behavior of the functions $f(\Lambda, L, R)$ in Eq. (2), which reflects the correlation properties of inhomogeneities, can vary from surface to surface even when the main correlation parameters $l$ and $R$ remain the same.

The correlation functions (1) are characterized by different long-range behavior that can be reliably identified in various surface diffraction and scattering experiments. What we would like to know is how sensitive is the particle transport to the form of the surface correlator. In contrast to surface diffraction and scattering data with angular and/or wavelength scanning, the transport coefficients are integral parameters that include angular and wavelength averaging. This leaves the question of how sensitive is the conductivity to the shape of the surface correlator wide open. In addition, we are asking a question whether it is possible to identify the type of surface inhomogeneities from transport experiments in ultrathin films or multilayer systems without prior information on the form of the surface correlator. The interrelated question is, of course, to what extent one should pay attention to the details of the correlator of surface inhomogeneities in analytical or numerical transport calculations for particles with large mean free paths. The former issue has already been raised in Refs. 3 and 4 for a small set of surface correlators on the basis of the Born approximation for wall scattering. Below we present a systematic study which is based on a more general transport formalism and involves a variety of classes of surface correlators.

In short, we want to compare functions $f(\Lambda, L, R)$ in Eq. (2) calculated for various types of the correlation functions $\zeta(s)$ in a wide range of parameters. We start from degenerate ballistic fermions in quantized metal films. The choice is not arbitrary: transport in such systems involves the minimal degree of averaging (integration) and can be the most sensitive to the long-range properties of the surface correlators (1).

The quantum size effect in metal films is a subject of intensive experimental study. Recent QSE experiments with quantized metal films include conductivity, ${ }^{5}$ spectroscopy, ${ }^{6}$ susceptibility, ${ }^{7}$ and scanning tunneling microscopy ${ }^{8}$ (STM) measurements. One of the signature features of the QSE in metals is a pronounced sawlike dependence of conductivity on, for example, film thickness $\sigma(L)$. This dependence was predicted for both bulk ${ }^{9}$ and surface ${ }^{10}$ scattering. Experimentally, the QSE in conductivity was studied for metals in Refs. 5 and 11 (for earlier results see references therein). However, experiments on the QSE in metals have to overcome a diffi- culty which one does not encounter in semiconductors. The period of the QSE oscillations in the dependence $\sigma(L)$ is usually small, almost atomic, $1 / p_{F}$ (below, except for final results, $\hbar=1$ ). For this reason typical experimental objects are lead or semimetal films such as bismuth. Below we predict a new type of QSE with large-period oscillations of $\sigma(L)$ at relatively large values of $p_{F} L$ that could lead to observation of a QSE in a wider group of metals. Largeperiod QSE oscillations have already been observed (see the second Ref. 5); however, sketchy experimental details do not allow one to identify reliably this observation as the new type of QSE predicted below. Our results can also resolve the long-standing controversy on the influence of the structure of the nanoscale film on its resistivity. ${ }^{11}$

Recently, we developed a transparent semianalytical formalism for transport in systems with rough boundaries that allows simple uniform calculations in a wide range of parameters and for various types of roughness with and without bulk scattering. ${ }^{12-14}$ This formalism unites approaches by Tesanovic et al., ${ }^{15}$ Fishman and Calecki, ${ }^{16}$ Kawabata, ${ }^{17}$ Meyerovich and S. Stepaniants, ${ }^{18}$ and Makarov et al. ${ }^{19}$ (for a brief comparison between different theoretical approaches see Refs. 13 and 20). Below we apply this formalism with an explicit purpose of studying the dependence of the transport coefficients on the shape of the correlation function of surface inhomogeneities. The well-defined limits of applicability of our approach to metal and semiconductor films are discussed in detail in Refs. 13 and 14.

Since the 2D mobility of particles is described by essentially the same equations as the exponent in the expression for the localization length in films, our study provides the dependence of the localization length on the type of the correlation function of random surface inhomogeneities.

The paper has the following structure. In the next section we introduce various types of surface correlation functions. Section III briefly describes the transport equation used for conductivity (mobility) calculations in QSE conditions. The results of transport calculations for different types of correlators are given in Sec. IV. Conclusions and experimental implications are discussed in Sec. V. Appendix A contains useful analytical expressions for the power density spectral functions of inhomogeneities responsible for the behavior of scattering probabilities for different types of correlators. Appendix B deals with the positions of new type of QSE peaks.

\section{CORRELATION FUNCTION OF SURFACE INHOMOGENEITIES}

We consider an infinite 2D channel (or film) of the average thickness $L$ with random rough boundaries

$$
x=L / 2-\xi_{1}(y, z), \quad x=-L / 2+\xi_{2}(y, z)
$$

(the walls are assumed hard with infinite potential). The inhomogeneities are small, $\xi_{1,2}(y, z) \ll L$, and random with zero average, $\left\langle\xi_{1}\right\rangle=\left\langle\xi_{2}\right\rangle=0$. Their correlation function $\zeta_{i k}(\mathbf{s})$ and its Fourier image $\zeta_{i k}(\mathbf{q})$, which is often called the power spectral density function or power spectrum, are defined as 


$$
\begin{aligned}
& \zeta_{i k}(|\mathbf{s}|)=\left\langle\xi_{i}\left(\mathbf{s}_{1}\right) \xi_{k}\left(\mathbf{s}_{1}+\mathbf{s}\right)\right\rangle \equiv A^{-1} \int \xi_{i}\left(\mathbf{s}_{1}\right) \xi_{k}\left(\mathbf{s}_{1}+\mathbf{s}\right) d \mathbf{s}_{1}, \\
& \zeta_{i k}(|\mathbf{q}|)=\int d^{2} s e^{i \mathbf{q} \cdot \mathbf{s}} \zeta_{i k}(|\mathbf{s}|)=2 \pi \int_{0}^{\infty} \zeta_{i k}(s) J_{0}(q s) s d s
\end{aligned}
$$

where $\mathbf{s}=(y, z)$ and $\mathbf{q}=\left(q_{y}, q_{z}\right)$ are the $2 \mathrm{D}$ vectors. In homogeneous systems, the correlation function depends only on the distance between points $\left|\mathbf{s}_{1}-\mathbf{s}_{2}\right|$ and not on coordinates themselves. The correlation functions $\zeta_{11}$ and $\zeta_{22}$ describe intrawall correlations of inhomogeneities and $\zeta_{12}=\zeta_{21}$ are the interwall correlations. Usually, but not always, the inhomogeneities on different walls are not correlated with each other, $\zeta_{12}=0$. Thus, everywhere, except for Sec. IV E, it is assumed that $\zeta_{12}=0$. To avoid parameter clutter, we also assume that the correlation parameters are the same on both walls, $\zeta_{11}=\zeta_{22}=\zeta$. Then the effective correlator contains $2 \zeta(s)$ with $\zeta(s)$ given by equations below.

Surface inhomogeneities exhibit a variety of types of the correlation functions. ${ }^{1,2}$ To have a meaningful comparison, we consider the correlation functions that involve only two characteristic parameters: namely, the amplitude (average height) $l$ and the correlation radius (average size) $R$ of surface inhomogeneities.

The most commonly used in theoretical applications is the Gaussian correlation function

$$
\zeta(s)=l^{2} \exp \left(-s^{2} / 2 R^{2}\right), \quad \zeta(q)=2 \pi l^{2} R^{2} \exp \left(-q^{2} R^{2} / 2\right),
$$

including its limit for small correlation radius $R \rightarrow 0$, i.e., the $\delta$-type correlations:

$$
\zeta(s)=l^{2} R^{2} \delta(s) / s, \quad \zeta(q)=2 \pi l^{2} R^{2} .
$$

Sometimes, a better fit to experimental data on surface scattering is provided by the use of the exponential correlation function

$$
\zeta(s)=l^{2} \exp (-s / R), \quad \zeta(q)=\frac{2 \pi l^{2} R^{2}}{\left(1+q^{2} R^{2}\right)^{3 / 2}},
$$

or by the even more long-range, power-law correlators

$$
\begin{gathered}
\zeta(s)=\frac{2 \mu l^{2}}{\left(1+s^{2} / R^{2}\right)^{1+\mu}}, \\
\zeta(q)=2 \pi l^{2} R^{2} \frac{(q R)^{\mu}}{2^{\mu-1} \Gamma(\mu)} K_{\mu}(q R)
\end{gathered}
$$

with different values of the parameter $\mu$. The most commonly used are the Staras function with $\mu=1$ and the correlator with $\mu=1 / 2$ which has the exponential power spec$\operatorname{trum} \zeta(q)$ :

$$
\zeta(q)=2 \pi l^{2} R^{2} \exp (-q R)
$$

The use of the Lorentzian correlator, which differs from the definition (8) at $\mu \rightarrow 0$ by the factor $\mu$ in the numerator,

$$
\zeta(s)=\frac{2 l^{2}}{1+s^{2} / R^{2}}, \quad \zeta(q)=2 \pi l^{2} R^{2} K_{0}(q R)
$$

deserves a special comment. This correlator is often considered as "unphysical." Its Fourier image (10) contains a function $K_{0}(q R)$ that diverges logarithmically at long wavelengths $q \rightarrow 0$. The issue to what extent the correlators are "physical" and can be reproduced experimentally is irrelevant in our context. For us, the fact that the Lorentzian correlator is sometimes used in calculations is sufficient enough to consider this correlator in the paper. To deal with the divergency, one can truncate the Lorentzian correlator at large distances (the common practice is to make a cut-off at the distances about 0.1 of the system length $\left.{ }^{1}\right)$. Another option is to use the generalized power-law correlator (8) with small $\mu$ instead of the Lorentzian (10). In order not to introduce additional parameters, we use the untruncated equation (10). Even though the divergence of $K_{0}(q R \rightarrow 0)$ does not lead to any singularities in transport coefficients, the transport coefficients for Lorentzian surfaces (see below) often behave qualitatively different from systems with other types of random inhomogeneities, even from the systems (8) with small $\mu$. [Sometimes, the divergence of the power spectrum $\zeta(q)$ is associated with the fractal nature of the surface, ${ }^{1}$ to what extent our transport formalism can be used for films with fractal surfaces is an open question.]

The last class of correlation functions covers the powerlaw correlators in momentum space:

$$
\zeta(q)=\frac{2 \pi l^{2} R^{2}}{\left(1+q^{2} R^{2}\right)^{1+\lambda}}, \quad \zeta(s)=l^{2} \frac{(s / R)^{\lambda}}{2^{\lambda} \Gamma(1+\lambda)} K_{\lambda}(s / R) .
$$

The correlators from this group include the Lorentzian in momentum space $\lambda=0$ that was observed in Ref. 2 (see also Ref. 4) and the exponential correlator (7) at $\lambda=1 / 2$.

The constants in all these correlators are chosen in such a way that the value of $\zeta(q=0)=2 \pi l^{2} R^{2}$ is the same. This provides a reasonable basis of comparison for transport coefficients in films with all these different types of random surfaces. Indeed, the scattering cross section for $q \rightarrow 0$ does not depend on the details of short-range and midrange structure of surface inhomogeneities. Therefore, at Fermi momenta $q_{F} \rightarrow 0$ (more precisely, at $q_{F} R \ll 1$ ), the transport coefficients should be the same for all random surfaces. [The only exception is the Lorentzian (10) for which $\zeta(q)$ diverges at small $q$.]

In what follows we compare the transport properties of the films (5)-(11) in various ranges of the film thickness $L$, correlation radius $R$, and particle wavelength $\Lambda_{F}=1 / q_{F}$ (or 2D particle density $N$ ).

\section{TRANSPORT EQUATION FOR BALLISTIC DEGENERATE FERMIONS IN QUANTIZED FILMS}

The QSE is caused by quantization of motion in the direction perpendicular to the film, $p_{x} \rightarrow \pi j / L$, and leads to a split of the energy spectrum $\epsilon(\mathbf{p})$ into a set of minibands, $\epsilon\left(p_{x}, \mathbf{q}\right) \rightarrow \boldsymbol{\epsilon}(\pi j / L, \mathbf{q})=\epsilon_{j}(\mathbf{q})$. For simplicity, we consider 
circular Fermi surfaces $\epsilon_{j}(\mathbf{q})=\epsilon_{F}$ :

$$
\begin{aligned}
\boldsymbol{\epsilon}_{j}(\mathbf{q})= & \frac{1}{2 m}\left[(\pi j / L)^{2}+q_{j}^{2}\right], \quad q_{j} \equiv q_{F j}=\left[2 m \epsilon_{F}\right. \\
& \left.-(\pi j / L)^{2}\right]^{1 / 2},
\end{aligned}
$$

where $q_{j}$ is the Fermi momentum for the miniband $j$. One can introduce the overall Fermi momentum as

$$
q_{F}=1 / \Lambda_{F}=\left(2 m \epsilon_{F}\right)^{1 / 2} .
$$

The relationship between this Fermi momentum $q_{F}$ and the 2D density of fermions $N_{2}$ in quantized films is somewhat cumbersome, ${ }^{12}$

$$
N=\sum N_{j}=(S / 2 \pi)\left[q_{F}^{2}-(\pi / L)^{2}(S+1)(2 S+1) / 6\right],
$$

where $S$ is the number of the occupied minibands:

$$
S=\operatorname{Int}\left[q_{F} L / \pi\right] .
$$

If the density of fermions is the same as in the bulk, then $N_{2}=n_{3} L$ where $n_{3}$ is the usual bulk density. Even in this case, the number of the occupied minibands $S$, according to Eqs. (14), (15), is a complicated function of L. Asymptotically, at large $S$,

$$
S=\operatorname{Int}\left[\left(3 N_{2} L^{2} / \pi\right)^{1 / 3}\right] .
$$

According to Refs. 12 and 13, scattering by random surface inhomogeneities results in intra- and interband transitions $\boldsymbol{\epsilon}_{j}(\mathbf{q}) \rightarrow \boldsymbol{\epsilon}_{j^{\prime}}\left(\mathbf{q}^{\prime}\right)$ with transition probabilities $W_{j j^{\prime}}\left(\mathbf{q}, \mathbf{q}^{\prime}\right)$ that are expressed explicitly via the surface correlation function $\zeta\left(\left|\mathbf{q}-\mathbf{q}^{\prime}\right|\right)$ :

$$
\begin{aligned}
W_{j j^{\prime}}\left(\mathbf{q}, \mathbf{q}^{\prime}\right)= & \frac{\hbar}{m^{2} L^{2}}\left[\zeta_{11}+\zeta_{22}+2 \zeta_{12}(-1)^{j+j^{\prime}}\right] \\
& \times\left(\frac{\pi j}{L}\right)^{2}\left(\frac{\pi j^{\prime}}{L}\right)^{2} .
\end{aligned}
$$

The generalization to other, more complicated energy spectra is straightforward. ${ }^{13}$

The transport equation for the distribution functions $n_{j}(\mathbf{q})$,

$$
\frac{d n_{j}}{d t}=2 \pi A \sum_{j^{\prime}} \int W_{j j^{\prime}}\left[n_{j^{\prime}}-n_{j}\right] \delta\left(\boldsymbol{\epsilon}_{j \mathbf{q}}-\epsilon_{j^{\prime} \mathbf{q}^{\prime}}\right) \frac{d^{2} q^{\prime}}{(2 \pi)^{2}},
$$

reduces, after standard transformations, to a set of linear equations

$$
\begin{gathered}
q_{j} / m=-\sum_{j^{\prime}} \nu_{j^{\prime}}\left(q_{j^{\prime}}\right) / \tau_{j j^{\prime}}, \\
\frac{2}{\tau_{j j^{\prime}}}=m \sum_{j^{\prime \prime}}\left[\delta_{j j^{\prime}} W_{j j^{\prime \prime}}^{(0)}-\delta_{j^{\prime} j^{\prime \prime}} W_{j j^{\prime}}^{(1)}\right],
\end{gathered}
$$

where $n_{j}^{(1)}=\nu_{j} \delta\left(\epsilon-\epsilon_{F}\right) e E$ is the first angular harmonic of the distribution function $n_{j}(\mathbf{q})$ at $q=q_{j}$, and $W_{j j^{\prime}}^{(0,1)}\left(q_{j}, q_{j^{\prime}}\right)$ are the zeroth and first harmonics of $W\left(\mathbf{q}_{j}-\mathbf{q}_{j^{\prime}}\right)$ over the angle $\widehat{\mathbf{q}}_{j} \mathbf{q}_{j^{\prime}}$. For some of the correlation function from the previous section the angular harmonics can be calculated analytically (see Appendix A). For others, this calculation is performed numerically. film:

The solution of Eqs. (19) provides the conductivity of the

$$
\sigma=-\frac{e^{2}}{3 \hbar^{2}} \sum_{j} \nu_{j}\left(q_{j}\right) q_{j}
$$

Equations (19) have simple analytical solution when the matrix $\tau_{j j^{\prime}}^{-1}$ can be approximated by a diagonal matrix, $\tau_{j j^{\prime}}^{-1}$ $\approx \delta_{j j^{\prime}} / \tau_{j}$ :

$$
\sigma=\frac{e^{2}}{3 \hbar^{2} m} \sum_{j} q_{j}^{2} \tau_{j}
$$

This happens when the matrix $W_{j j^{\prime}}^{(1)}$ is almost or exactly diagonal, $W_{j j^{\prime}}^{(1)} \simeq W_{j}^{(1)} \delta_{j j^{\prime}}$ and

$$
2 / m \tau_{j}=\sum_{j^{\prime}} W_{j j^{\prime}}^{(0)}-W_{j}^{(1)} .
$$

Then the conductivity (21) is equal to

$$
\sigma=\frac{e^{2}}{3 \hbar^{2} m} \sum_{j} \tau_{j} q_{j}^{2}=\frac{2 e^{2}}{3 \hbar^{2} m^{2}} \sum_{j} \frac{q_{j}^{2}}{\sum_{j^{\prime}} W_{j j^{\prime}}^{(0)}-W_{j}^{(1)}} .
$$

Such a diagonalization occurs in three physical situations. The simplest one is the one when only one miniband is occupied and

$$
\sigma=\frac{e^{2}}{3 \hbar^{2} m} \tau_{1} q_{1}^{2}=\frac{2 e^{2} q_{1}^{2}}{3 \hbar^{2} m^{2}} \frac{1}{W_{11}^{(0)}-W_{11}^{(1)}} .
$$

The second case is the case of systems with large correlation length $R \gg L$. In such systems the intraband scattering is much stronger than the interband one and the off-diagonal matrix elements $W_{j j^{\prime}}$ are small in comparison with the diagonal ones (see Appendix A). Then both matrices $W_{j j^{\prime}}^{(0,1)}$ are almost diagonal,

$$
W_{j j^{\prime}}^{(0,1)} \simeq W_{j}^{(0,1)} \delta_{j j^{\prime}},
$$

and the expression for the conductivity, Eq. (23), reads

$$
\sigma \simeq \frac{2 e^{2}}{3 \hbar^{2} m^{2}} \sum_{j} \frac{q_{j}^{2}}{W_{j}^{(0)}-W_{j}^{(1)}}
$$

Such diagonalization of the matrices $W_{j j^{\prime}}^{(0,1)}(25)$ at $R \gg L$ can often be an oversimplification (see Sec. IV). 
The third situation with diagonal $\tau_{j j^{\prime}}^{-1}$ is the case of small $q R$. In this limit, the correlation function is a constant with zero first harmonic:

$$
W_{j j}^{(0)}=2 W(q R \rightarrow 0), \quad W_{j j}^{(1)}=0 .
$$

According to Eq. (17),

$$
W_{j j^{\prime}}(0)=\frac{2 \hbar}{m^{2} L^{2}} \zeta(0)\left(\frac{\pi j}{L}\right)^{2}\left(\frac{\pi j^{\prime}}{L}\right)^{2}
$$

and

$$
\sigma=\frac{2 e^{2}}{\hbar} \frac{(L / \pi)^{4}}{2 S(S+1)(2 S+1) \zeta(0)} \sum_{j}\left(\frac{L q_{j}}{\hbar j}\right)^{2} .
$$

Note that all our surface correlators $\zeta(s)$ are introduced in such a way that in the long-wave limit $\zeta(q \rightarrow 0)$ they are, except for the Lorentzian (10), equal to each other, $\zeta(0)$ $=2 \pi l^{2} R^{2}$. This means that in this limit the conductivities (28) are the same irrespective of the shape of the correlator:

$$
\sigma=\frac{2 e^{2}}{\hbar} \frac{1}{4 \pi} \frac{\left(L^{2} / \pi^{2} l R\right)^{2}}{S(S+1)(2 S+1)} \sum_{j}\left(\frac{L q_{j}}{\hbar j}\right)^{2}
$$

(cf. Ref. 16).

In all other situations Eqs. (19) are not diagonal and should be solved numerically.

The results for conductivity (mobility) also provide the exponent in the expression for the localization length $\mathcal{R}$ that describes localization caused by particle scattering by random wall inhomogeneities: ${ }^{13}$

$$
\mathcal{R}=\mathcal{L} \exp [\pi m S D / \hbar]
$$

where $\mathcal{L}$ is the mean free path and the diffusion coefficient $D$ is proportional to the conductivity $\sigma$.

\section{RESULTS AND DISCUSSION}

\section{A. General comments}

As is mentioned in the Introduction, the $2 \mathrm{D}$ conductivity $\sigma$ of the film has the dimensionality of conductance and is described by a dimensionless function $f$ in Eq. (2). This function, in turn, depends on the relation between three length scales: particle Fermi wavelength $\Lambda_{F}=1 / q_{F}$, width of the channel $L$, and correlation radius of the surface inhomogeneities $R$. The fourth length parameter $l$ is perturbative and enters the conductivity as a coefficient:

$$
\sigma=\frac{2 e^{2}}{\hbar} \frac{L^{2}}{l^{2}} f\left(\Lambda_{F}, L, R\right) .
$$

Note that we consider only the contribution from surface roughness and disregard bulk scattering. As a result, the conductivity (31) diverges in the limit of vanishing inhomogeneities $l \rightarrow 0$ or $R \rightarrow \infty$. The proper account of bulk scattering ${ }^{14}$ eliminates this divergence.

The dimensionless function $f\left(\Lambda_{F}, L, R\right)$ depends only on the ratio of these three lengths. Of three ratios $z=L / \Lambda_{F}$
$=q_{F} L, x=R / \Lambda_{F}=q_{F} R$, and $y=R / L=x / z$ only two are independent, $x=y z$. Which two of these ratios should be used as independent dimensionless variables depends on whether one wants to display the dependence of $\sigma$ on $\Lambda_{F}, L$, or $R$. The study of the dependence of the conductivity on film thickness, $\sigma(L)$, should be performed at constant $\Lambda_{F}$ and $R$. This means that $\sigma(L)$ is best displayed by the function $f_{L}(z, x)$,

$$
\sigma(L)=\frac{2 e^{2}}{\hbar} \frac{R^{2}}{l^{2}} f_{L}(z, x=\text { const }),
$$

for various values of $x=R / \Lambda_{F}$.

Plots of the function $f_{R}(y)$ at constant values of $z$ $=q_{F} L$,

$$
\sigma(R)=\frac{2 e^{2}}{\hbar} \frac{L^{2}}{l^{2}} f_{R}(y, z=\text { const }),
$$

reflect the dependence $\sigma(R)$. Similarly, plots of the function $f_{N}(z)$ at constant $y=R / L$,

$$
\sigma\left(q_{F}\right)=\frac{2 e^{2}}{\hbar} \frac{L^{2}}{l^{2}} f_{N}(z, y=\text { const }),
$$

characterize the dependence of conductivity on density of particles $N$ or the Fermi momentum $q_{F}$.

Below we compare these dimensionless functions, $f_{L}(z)$, $f_{R}(y)$, and $f_{N}(z)$ for various types of correlation functions in wide ranges of parameters. Needless to say, the results at $x$ $\rightarrow 0$ should coincide for all types of correlators except, maybe, for the Lorentzian, since, by design, all the correlation functions are the same in this limit [see Eq. (29)].

Curves in all figures below are labeled in a uniform way by the type of surface correlator used in calculations. Curves $G$ correspond to Gaussian inhomogeneities (5), Curves $L$ describe the surfaces with Lorentzian correlations (10); curves $\mu_{1}, \mu_{5}$, and $\mu_{9}$ give the results for the correlators (8) with $\mu=0.1,0.5,0.9$; and curves $\lambda_{0}, \lambda_{5}$, and $\lambda_{9}$ correspond to Eq. (11) with $\lambda=0,0.5,0.9$. Note that correlator $\mu_{5}$ has the exponential power spectrum (9) and that correlator $\lambda_{5}$ is actually the exponential correlator (7).

\section{B. Dependence on the film thickness}

Figures 1 and 2 for the function $f_{L}(z, x=$ const), Eq. (32), show the dependence of the conductivity $\sigma(L)$ for two different values of $R / \Lambda_{F}, x=1,10$, for various types of the correlation functions. The labeling of the curves $G, L, \mu_{1}, \mu_{5}, \mu_{9}, \lambda_{0}, \lambda_{5}, \lambda_{9}$ is explained at the end of the previous subsection. The main feature of the curves-namely, their sawlike character-is well known. The sharp drops occur when the number of the occupied minibands, Eq. (15), changes by 1, i.e., in the points $z=L / \Lambda_{F}=k \pi$ with integer $k$. The only unexpected feature is a "wrong" periodicity of the initial part of the Gaussian curve $G$ at small values of $z$ for $x=10$ (see the inset in Fig. 2). This feature will be explained later. The Lorentzian curve $L$ is different from others: at $x$ $=10$ the curve has already lost its QSE structure. 


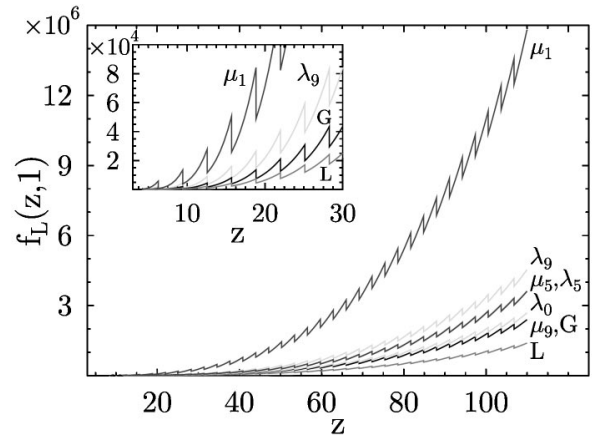

FIG. 1. Function $f_{L}\left(z, x=\right.$ const), Eq. (32), at $x=R / \Lambda_{F}=1$ for various correlation functions. The labeling of the curves is explained at the end of Sec. IV A. Curve $G$ : Gaussian correlator (5). Curves $\mu_{1}, \mu_{5}, \mu_{9}$ : power-law correlators (8) with $\mu=0.1,0.5,0.9$. Curve $L$ : Lorentzian correlator. Curves $\lambda_{0}, \lambda_{5}, \lambda_{9}$ : power-law correlators in momentum space (11) with $\lambda=0,0.5,0.9[\lambda=0.5$ corresponds to the exponential correlator in the coordinate space (7)]. The sharp drops occur when the number of the occupied minibands $S$, Eq. (15), changes by 1, i.e., in the points $z=L / \Lambda_{F}=k \pi$ with integer $k$.

At these relatively small values of $x$, the curves for all types of correlators have roughly the same shape though the exact values of the conductivity are different. (Curves $\mu_{5}$ and $\lambda_{5}$ are indistinguishable in both Figs. 1 and 2, and curves $G$ and $\mu_{9}$ are indistinguishable in Fig. 1.) To underscore this point, in Figs. 3 and 4 we plotted instead of the curves $f_{L}(z)$ the normalized curves $f_{L}(z) / f_{L}\left(z=z_{\max }\right)$ with the normalization coefficients ensuring that the values of the normalized conductivity are equal to 1 at the highest values of $z$ in the plot. Strikingly, for $x=1$ (Fig. 3) all the normalized curves with these eight correlation functions lie within one bold line and are all indistinguishable with this resolution. For larger $x$, the difference is still insignificant: all the curves are compressed between curves $G$ and $L$. The only anomaly is the loss of QSE structure by curve $L$.

The main conclusion here is that the shape of the dependence $\sigma(L)$ at constant $R$ and $q_{F}$ is not sensitive to and cannot provide any information on the type of the correlator at not very large values of $R / \Lambda_{F}$. Since $l$ is unknown and enters the conductivity as a coefficient, the absolute values of $\sigma(L)$ cannot serve as a clue either: experimental data on

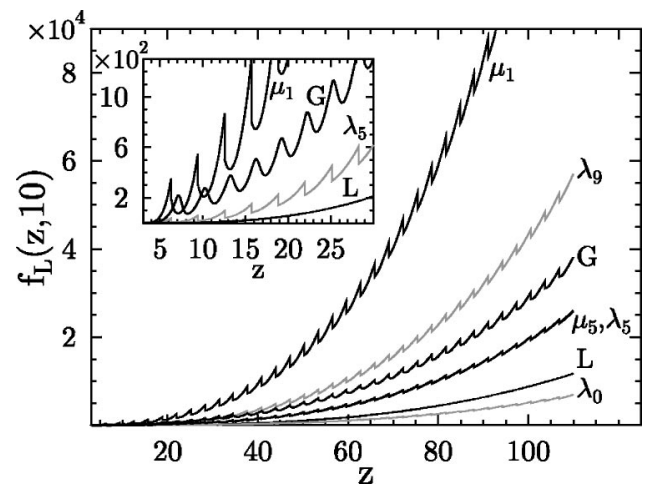

FIG. 2. The same as in Fig. 1 for $x=10$. The labeling of the curves is explained at the end of Sec. IV A.

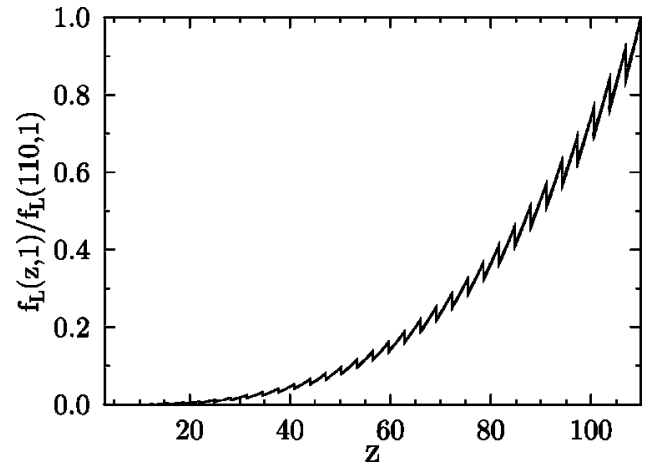

FIG. 3. The same eight functions $f_{L}(z, x=1)$ as in Fig. 1 normalized by their value at $z=110, f_{L}(z) / f_{L}(110)$. All eight normalized curves are indistinguishable. The normalization coefficients are curve $G, f_{L}(110)=2.4 \times 10^{6}$; curve $L, f_{L}(110)=1.39 \times 10^{6}$; curve $\mu_{1}, f_{L}(110)=1.48 \times 10^{7}$; curve $\mu_{5}, f_{L}(110)=3.61 \times 10^{6}$; curve $\mu_{9}, f_{L}(110)=2.42 \times 10^{6}$; curve $\lambda_{0}, f_{L}(110)=2.69 \times 10^{6}$; curve $\lambda_{5}, f_{L}(110)=3.65 \times 10^{6}$; curve $\lambda_{9}, f_{L}(110)=4.54 \times 10^{6}$.

$\sigma(L)$ at moderate $R / \Lambda_{F}$ can be fitted by any type of the correlator by a choice of $l$. In this case, it is impossible to make any conclusion on the type of correlation function from transport measurements and it does not matter what correlator to use in theoretical calculations. Meaningful analysis requires some beforehand information on the correlation parameters. The only correlator that can be identified is the Lorentzian; however, this type of correlation is the least probable and might be "unphysical."

The situation changes dramatically at higher $x=R / \Lambda_{F}$ as is shown in Figs. 5 [function $f_{L}(z, x=400)$ ] and Fig. 6 [normalized function $\left.f_{L}(z, x=400) / f_{L}\left(z=z_{\max }, x=400\right)\right]$ for the same eight correlators (the labeling of the curves is explained in the end of Sec. IV A).

We anticipated one feature: namely, the decrease in the amplitude of sawteeth with increasing $x$ and even the disappearance of such teeth for the Gaussian correlator. The sharp drops in conductivity in the points where the number of the

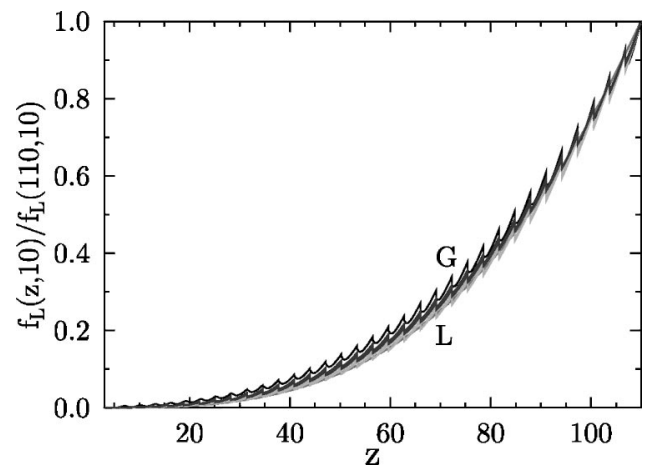

FIG. 4. The same eight functions $f_{L}(z, x=10)$ as in Fig. 2 normalized by their value at $z=110, f_{L}(z) / f_{L}(110)$. All eight curves lie between normalized curves $G$ and $L$ and are barely distinguishable. The normalization coefficients are, curve $G, f_{L}(110)=3.82$ $\times 10^{4}$; curve $L, f_{L}(110)=1.17 \times 10^{4}$; curve $\mu_{1}, f_{L}(110)=1.48$ $\times 10^{5}$; curve $\mu_{5}, f_{L}(110)=2.59 \times 10^{4}$; curve $\mu_{9}, f_{L}(110)=1.32$ $\times 10^{4}$; curve $\lambda_{0}, f_{L}(110)=6.95 \times 10^{3}$; curve $\lambda_{5}, f_{L}(110)=2.61$ $\times 10^{4}$; curve $\lambda_{9}, f_{L}(110)=5.7 \times 10^{4}$. 


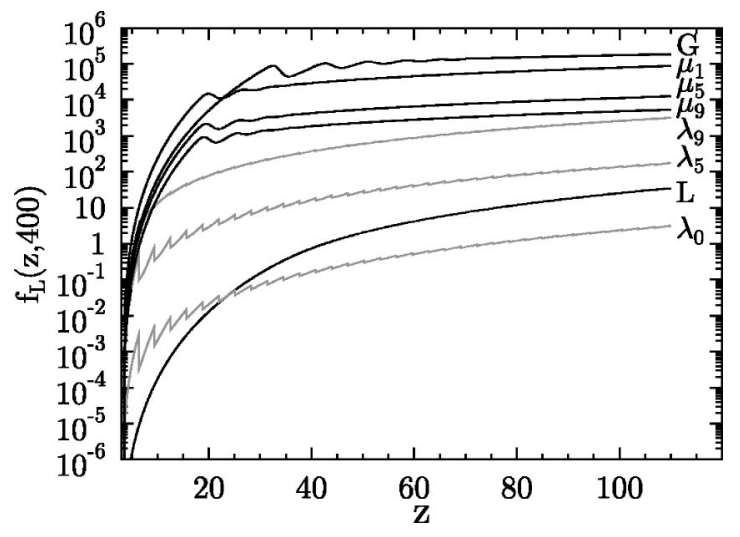

FIG. 5. Functions $f_{L}(z, x=400)$ for the same eight types of correlators as in Fig. 1.

occupied minibands $S$ increases by 1 is explained by opening of $S$ new scattering channels associated with interband transitions in and from this newly opened miniband. Without the interband transitions, the increase of $S$ by 1 results not in a sharp drop in $\sigma$, but in an insignificant kink on the curve $\sigma(L)$ as it is shown in the third reference of Ref. 18. The interband transitions are described by the off-diagonal components of the matrix of transition probabilities $W_{j j^{\prime}}$. With increasing $R / \Lambda_{F}$, these off-diagonal (interband) transition probabilities go to zero though with different rate for different types of the correlation function. The rate of decrease of the interband transition probabilities as a function of $R / \Lambda_{F}$ for different correlation functions is discussed in Appendix A. This rate is a good predictor for observing the sawlike shape of $\sigma(L)$. The fastest decrease happens in the case of the Gaussian correlator; thus the curve for the Gaussian correlator should be the smoothest and should exhibit the smallest traces of the sawteeth. Therefore, the visibility of the sawteeth on the experimental curve can be a clue to the form of the correlation function.

What is completely unexpected is the appearance of a new type of oscillation structure on $\sigma(L)$ in a limited range

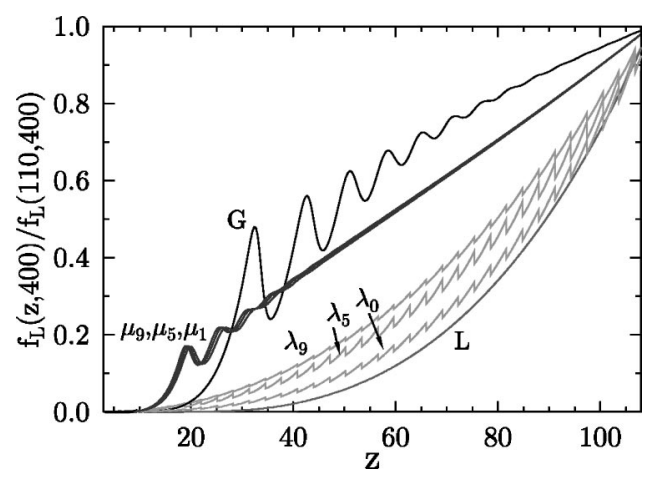

FIG. 6. The same eight functions $f_{L}(z, x=400)$ as in Fig. 5 normalized by their value at $z=110, f_{L}(z) / f_{L}(110)$. The normalization coefficients are curve $G, f_{L}(110)=1.84 \times 10^{5}$; curve $L$, $f_{L}(110)=35.0 ; \quad$ curve $\mu_{1}, f_{L}(110)=8.78 \times 10^{4} ;$ curve $\mu_{5}$, $f_{L}(110)=1.25 \times 10^{4} ;$ curve $\mu_{9}, f_{L}(110)=5.35 \times 10^{3}$; curve $\lambda_{0}$, $f_{L}(110)=3.16$; curve $\lambda_{5}, f_{L}(110)=1.76 \times 10^{2}$; curve $\lambda_{9}, f_{L}(110)$ $=3.21 \times 10^{3}$. of $z$ for the Gaussian and power-law correlators (curves $G$ and $\mu_{i}$ in Figs. 5 and 6). It looks as if there is a transition between two distinct regimes with several sharp oscillations in the transition range. The effect looks even more striking in Fig. 6 for the normalized curves which, in contrast to Fig. 5, are plotted in a linear scale. This new type of QSE requires an explanation.

These new oscillations are not related to abrupt changes in the number of occupied minibands $S(z)$ : the oscillations are less sharp, have a much larger period, and, most important, appear only in a limited range of $z$ where the number of occupied minibands $S$ is already large. These new oscillations are observed for the correlators for which the interband transitions are the smallest and the sawlike structure is suppressed-namely, for the Gaussian and power-law correlation functions. The power spectrum for these correlators $\zeta(q)$ goes to zero exponentially at large $q$. Then one would expect that the off-diagonal (interband) transition probabilities are exponentially small in comparison with intraband scattering and that the conductivity can be well described by the "diagonal" approximation (26) that does not have an oscillation feature. This turns out not to be the case.

The oscillations are indeed related to off-diagonal (interband) scattering probabilities $W_{j j^{\prime}}$. A qualitative explanation of the effect and an estimate of the peak positions are the following. Scattering by surface inhomogeneities changes the tangential momentum by $\Delta q \sim 1 / R$. According to the momentum conservation law, this scattering can cause the interband transition $j \leftrightarrow j+1$ only when $q_{j}-q_{j+1}=\Delta q$ $\sim 1 / R$. If the miniband index $j$ is relatively small and $q_{j}$ $\sim 1 / \lambda_{F}$, then $q_{j}-q_{j+1} \sim\left(q_{j}^{2}-q_{j+1}^{2}\right) \lambda_{F} / 2$. The energy conservation requires that $q_{j}^{2}-q_{j+1}^{2}=\pi^{2}(j+1)^{2} / L^{2}-\pi^{2} j^{2} / L^{2}$ $\sim 2 \pi^{2} j / L^{2}$. The combination of these conservation laws defines the peak positions $L_{j}$, which correspond to the opening of robust interband transitions $j \leftrightarrow j+1$ and which are given by equations $L_{j}^{2} \sim \pi^{2} j R \lambda_{F}$. In dimensionless variables, this is equivalent to

$$
z_{j} \sim \pi \sqrt{j x}
$$

Accordingly, with increasing film thickness $L$ the transition channel opens first for the electrons in the lowest miniband $\epsilon_{1}(\mathbf{q})$ with $j=1$. Note, that these are the grazing electrons which are responsible for the dominant contribution to the conductivity. Thus, the conductivity drops almost by half at the film thickness $z_{1} \sim \pi x^{1 / 2}$ where $W_{12}$ becomes comparable to $W_{11}$ and the effective cross-section doubles. At higher value of $L, z_{2} \sim \pi(2 \pi)^{1 / 2}$, a new channel $W_{23}$ opens to the electrons from the next miniband $j=2$ with $p_{x}$ $=2 \pi / L$ and the conductivity drops again, and so on. The only difference is that the contribution of the electrons from the higher minibands falls rapidly with an increase in the band index $j$ and the drops in conductivity $\sigma(L)$, which are associated with the opening of new scattering channels for electrons from these minibands, become smaller and smaller. The number of the visible peaks on the curve $\sigma(L)$ and their relative heights give a good visual estimate of the number of "important" minibands and of their relative contribution to the conductivity. With further increase in the film thickness, when $L$ becomes larger, $L \gg R$, the change of momentum 
$\Delta g \sim 1 / R$ is sufficient to excite all interband transitions and the ordinary QSE with the saw teeth at the points $z \sim \pi j$ is restored.

The above explanation works for the films with the exponential decay of the power spectrum of inhomogeneities in which the size of inhomogeneities $R$ is well-defined. In the films with a non-exponential power spectrum of inhomogeneities, i.e, with a more uniform distribution of inhomogeneities over the size $R$ in momentum space, this new size effect cannot be observed because the particles from all minibands can always find the inohomogeneities of the right size that ensure the interband transitions irrespective of what is the separation between the walls.

More accurate explanation is the following. The offdiagonal $W_{j j^{\prime}}$ is a function of

$$
\nu_{j j^{\prime}}=\left|q_{j} R / \hbar-q_{j^{\prime}} R / \hbar\right|=x\left|\sqrt{1-(\pi j / z)^{2}}-\sqrt{1-\left(\pi j^{\prime} / z\right)^{2}}\right|
$$

and rapidly decreases with increasing $\nu_{j j^{\prime}}$ (see Appendix A). In general, the off-diagonal $\nu_{j j^{\prime}}$ is large at large $R$ (or $x$ ) while the diagonal elements $\nu_{j j}=0$. However, for large $z$ (large $S$ ) some of the elements $\nu_{j j^{\prime}}$ with small $j$, which are close to the main diagonal, could become small even for large $x$ :

$$
\nu_{j, j+1}(j+1 \ll z / \pi) \sim \frac{\pi^{2} x}{2 z^{2}}(2 j+1)
$$

$[j$ changes from 1 to $\operatorname{Int}(z / \pi)]$. Then at large $z$ the transitions $j \leftrightarrow j+1$ can become noticeable and Eqs. (19) become coupled. This coupling changes the solution of transport equation and, therefore, conductivity. According to Eqs. (19) the coupling between the minibands $j$ and $j+1$ becomes noticeable, $\tau_{j, j+1}^{-1} \sim \tau_{j j}^{-1}$, when

$$
W_{j, j+1}^{(0)}(x, z) \sim W_{j j}^{(0)}(x, z)-W_{j j}^{(1)}(x, z) .
$$

At fixed $x$, Eq. (37) can be considered as the equation for the values of $z=z_{j}(x)$ at which one can observe the opening of transitions $j \leftrightarrow j+1$. The opening of such transition channels is accompanied by drops in conductivity. Since for the Gaussian and power-law correlators the interband transition probabilities $W_{j j^{\prime}}$ depend exponentially on parameters $\nu_{j j^{\prime}}$, these drops in conductivity are sharp and deep as illustrated in Figs. 5 and 6. Solutions $z_{j}(x)$ of Eqs. (37) are discussed in Appendix B. At $z=z_{1}(x), W_{12}$ is the first of transition probabilities to acquire the "normal" order of magnitude. At $z$ $=z_{2}(x), W_{23}$ becomes noticeable, then $W_{34}$, etc. The amplitudes of the drops rapidly decrease with increasing $j$. In the end, when several interband channels with $j \ll z / \pi$ are open, $\sigma(L)$ becomes smooth, but with a much lower slope than in its initial part. The growth of transition probabilities for transitions $j \leftrightarrow j+2$ does not result in new oscillations in $\sigma(L)$. In the points $z(x)$ where $W_{j, j+2}$ becomes large, $W_{j, j+2}^{(0)}$ $\sim W_{j j}^{(0)}-W_{j j}^{(1)}$, the states $j$ and $j+2$ are already strongly coupled via $W_{j, j+1}$ and $W_{j+1, j+2}$.

According to Appendix B, Eq. (B4), the positions of the drops for films with Gaussian surface inhomogeneities are similar to Eq. (35):

$$
z_{j}(x) \approx \frac{\pi}{2} \sqrt{(2 j+1) x}\{\ln [x \sqrt{2}(1+1 / j)]\}^{-1 / 4}
$$

The values $z_{j}(x=400)=33.4,43.6,51.8,58.9, \ldots$ agree well with the positions of the conductivity drops on curve 1 of Figs. 5 and 6.

For the surface with power-law correlations of inhomogeneities (8) the solution of Eq. (37) with logarithmic accuracy [Appendix B, Eq. (B9)] also resembles Eq. (35):

$$
\begin{gathered}
z_{j}(x)=\pi \sqrt{(2 j+1) x / 4 \nu}, \\
\nu \sim \ln \left(x(1+1 / j)\{2 \ln [x(1+1 / j)]\}^{\mu / 2+1 / 4}\right) .
\end{gathered}
$$

This expression is barely sensitive to $\mu$. This almost complete independence of the peak positions from $\mu$ can be clearly seen in Fig. 6.

The difference between this new type of size effect and the usual sawlike QSE is dramatic. The sawlike drops in conductivity for the usual QSE occur in the points $z=k \pi$ with integer $k$ and are a direct consequence of the quantization of momentum in thin films. The interband transitions are not germane to the existence or positions of this QSE and are responsible only for the amplitude of the conductivity oscillations. The drops in conductivity are equidistant with period $\pi$ along the $z$ axis, i.e., are equidistant as a function of film thickness. In contrast to this, the new QSE oscillations in Figs. 5 and 6 are not related directly to the quantization of momentum and are a consequence of the exponential opening of interband transitions between minibands with small quantum numbers at certain values of the film thickness. The transitions in and out of higher minibands remain suppressed. (In some sense, the effect resembles magnetic breakthrough between separated parts of the Fermi surface in high magnetic fields.) The peaks are roughly equidistant if plotted against $z^{2}$; weak deviation from periodicity is due to logarithmic terms in Eqs. (38) and (39). The period of the new QSE is much larger than for the usual QSE. The large period of oscillations can open the way to direct observation of the QSE in transport measurements in metal films in which the usual QSE has atomic period and can hardly be observed. There is a strong possibility that the conductivity oscillations reported in the last reference of Ref. 5 are actually this new type of QSE.

The initial part of the curves $G, \mu_{i}$ in Figs. 5 and 6 for $\sigma(L)$ is described analytically by Eq. (26) with appropriate values of $W$ from Appendix A. This curve is close to the power law $\sigma \propto L^{(5+\alpha)}$ (small $\alpha$ depends on $x$ ) and to experimental data of the third reference of Ref. 11. After the region of new QSE oscillations, the curves are again smooth, but with a much smaller tangent. We do not have an analytical description for this regime. The numerical approximation can be done equally well by either $\sigma=A+B L^{1+\beta}$ with small $\beta$ ( $\beta$ also depends on $x$ ) or a quadratic expression $a+b L$ $+c L^{2}$. This behavior explains the experimental data ${ }^{21}$ and the last Ref. 5. As a result, the power-law dependence of $\sigma(L)$ is qualitatively different for ultrathin and more thicker 


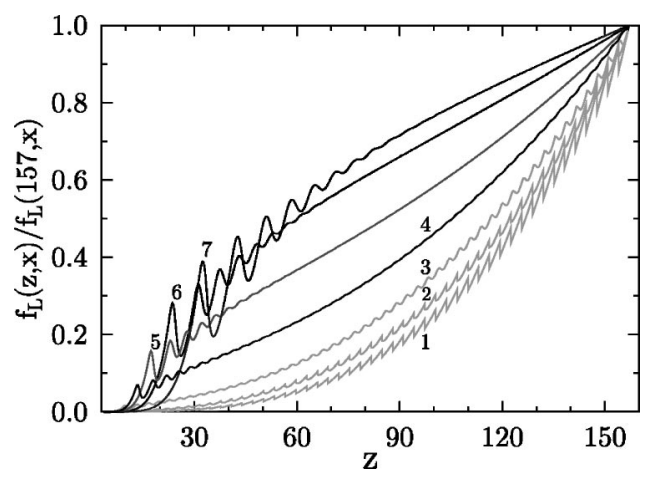

FIG. 7. Functions $f_{L}(z, x=$ const $)$ for Gaussian correlation of surface inhomogeneities normalized by their value at $z=157$, $f_{L}(z) / f_{L}(157)$. The values of $x$ and normalization coefficients are curve $1, x=1, f(157)=6.9 \times 10^{6}$; curve $2, x=10, f(157)=9.9$ $\times 10^{4}$; curve $3, x=25, f(157)=4.6 \times 10^{4}$; curve $4, x=55, f(157)$ $=3.8 \times 10^{4} ;$ curve $5, x=100, f(157)=4.75 \times 10^{4}$; curve $6, x$ $=200, f(157)=9.1 \times 10^{4}$; curve $7, x=400, f(157)=2.3 \times 10^{5}$.

films. This type of behavior is different from the earlier studied behavior of $\sigma(L)$ at small $x=q_{F} R \ll 1$. $^{3,13,16}$

The initiation of this new type of oscillations with a large period can be seen on the initial part of curve $G$ in Fig. 2 for $x=10$. With growing $z$ these new oscillations are overtaken by the standard QSE. The transition from standard to the new QSE is illustrated in Fig. 7 that contains normalized "curves $G$ " for the Gaussian inhomogeneities, $f_{L}(z, x=$ const $) / f_{L}(z$ $=157$ ), for $x=1,10,25,55,100,200,400$. It is clear from these curves how the usual QSE is replaced by new oscillations with increasing $x$. The "transitional" curve for $x=55$ is especially interesting: it shows the new QSE at smaller $z$ and a restoration of the standard QSE at higher $z$. This restoration occurs when a noticeable number of interband transitions become open at higher $z$. It seems that such a restoration does not happen on curves $x>50$. This impression is wrong. Such a restoration indeed occurs for curves $x=100,200,400$, but at values of $z$ that are much larger than those in the figure. At very large $x$, all curves $f_{L}(z, x=$ const $)$ consist of four parts: a rapid increase at small $z$, region of new QSE oscillations, smooth monotonic part, and region of relatively smooth standard QSE oscillations at the largest values of $z$. With increasing $x$, the amplitude of new QSE oscillations and the length of region separating new and old QSE increase rapidly.

\section{Dependence on the correlation radius}

The dependence of the conductivity on the correlation radius of surface inhomogeneities, $\sigma(R)$, is best illustrated by the function $f_{R}(y, z=$ const $)$, Eq. (33). Since the number of the occupied minibands $S$ does not depend on the correlation radius of inhomogeneities, the curves $f_{R}(y)$ at constant $z$ do not exhibit the sawlike structure. Instead, the two main features are the presence of the minimum in $f_{R}(y)$ and the steplike structure that corresponds to the oscillations in Figs. 5 and 6.

The scattering of fermions by surface inhomogeneities is most effective at $R / \Lambda_{F} \sim 1$, i.e., at $y \sim 1 / z$. This leads to a

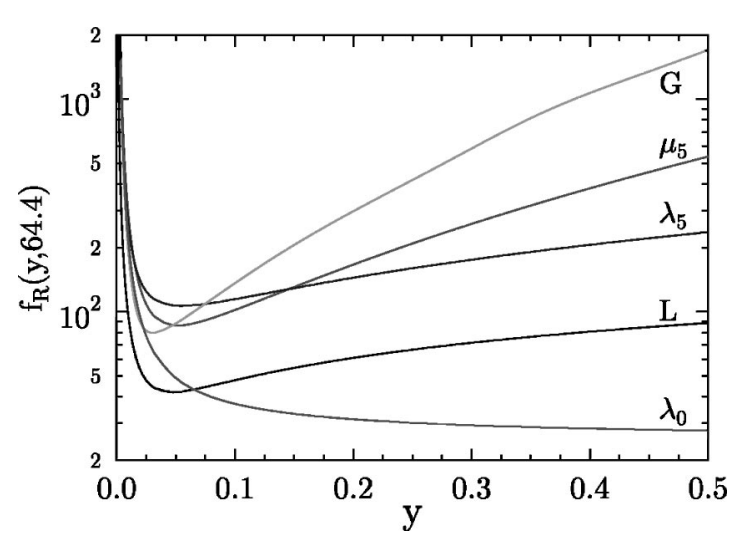

FIG. 8. Function $f_{R}(y, z=64.4)$, Eq. (33), near the minimum at $y z \sim 1$ for various surface correlators. The labeling of the curves is explained at the end of Sec. IV A.

minimum of the conductivity $\sigma(R)$ at such values of $y$. At $R / \Lambda_{F} \ll 1$ the particle wavelength is much larger than the size of surface inhomogeneities and the scattering is almost specular and does not contribute to the formation of the mean free path. In the opposite limit $R / \Lambda_{F} \gg 1$ the walls are flat on the particle length scale and surface scattering also does not limit the effective mean free path. Therefore, at $z$ $=$ const the conductivity $\sigma(R)$ for nondivergent correlators is infinite in both limits $y \rightarrow 0$ and $y \rightarrow \infty$ with a minimum around $y \sim 1 / z$. The curves $f_{R}(y)$ close to this minimum are plotted for different correlators in Fig. $8(z=64.4$; the labeling of the curves is explained in the end of Sec. IV A). It is important that the position of the minimum, its width, and even the order of magnitude of the function $f_{R}(y)$ in the minimum are roughly the same for all types of surface correlators. This is, probably, the most universal feature of the system. The only correlator that does not display a welldefined minimum is Eq. (11) with $\lambda=0$ (the Lorentzian in momentum space, curve $\lambda_{0}$ ). This feature is related to the logarithmic divergence of this correlator in "real" space. This feature is especially interesting because the surfaces with such inhomogeneities were observed in experiment. ${ }^{2}$

The drops in $\sigma(L)$ at large $z=z_{j}(x)$, which are analyzed in the previous section (Figs. 5 and 6), correspond to the points $y_{j}(z)$ on the curves $f_{R}(y)$. The positions of these points $y_{j}(z)$ are implicitly determined by Eqs. (38) and (39) for the Gaussian and power-law correlations provided that $x=y z$. These values of $y$ are far away to the right from the minimum in the curves $\sigma(R)$ and cannot be presented in the same figures. The feature that corresponds to the oscillations from the previous section is clearly seen as a set of steps in Fig. 9 for the same value of $z$ as in Fig. 8, $z=64.4$ on curves $G$ and $\mu_{5}$ for Gaussian and power-law inhomogeneities. For the surfaces with the Gaussian inhomogeneities, the first interband transition $W_{12}$ becomes visible for $z=64.4$ at $y_{1}$ $\sim 25$, the next one at $y_{2} \sim 14$, and so on. At these values of $y$ one can see well-pronounced steps on the curve $G$ in Fig. 9. The same feature, though barely discernible, is also observed for the power-law correlator $\mu_{5}$.

For comparison, curves $L, \lambda_{0}$, and $\lambda_{5}$ do not exhibit any anomalies. Interestingly, the curve for the Lorentzian inho- 


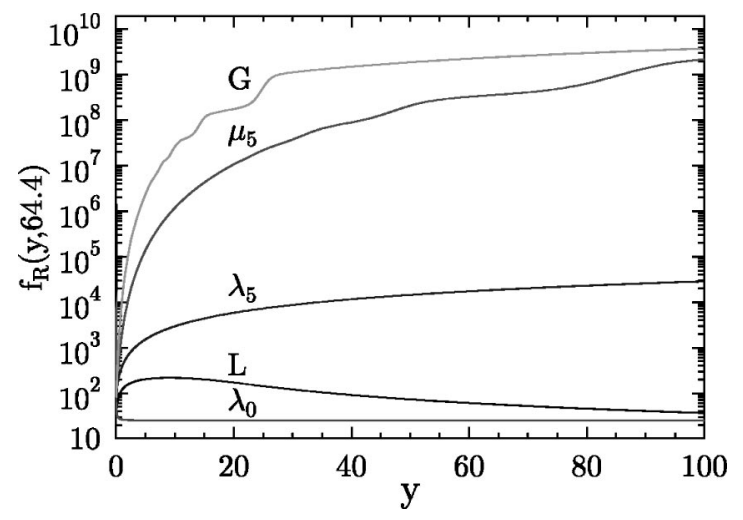

FIG. 9. The same functions $f_{R}(y, z=64)$, Eq. (33), as in Fig. 8 at larger values of $z$. The labeling of the curves is explained at the end of Sec. IV A.

mogeneities is the only one that decreases with increasing $y$ after the initial increase at small $y$ (Fig. 8). How this feature is related to the peculiarities of the Lorentzian that have been discussed in Sec. II is unclear. The curve $\lambda_{0}$ remains essentially flat.

\section{Dependence on the Fermi momentum and density of fermions}

The dependence of the conductivity $\sigma$ on the density of fermions, $N$, or their Fermi momentum $q_{F}$ is best displayed by the function $f_{N}(z)$ at constant $y=R / L$; see Eq. (34). This dependence $\sigma(N)$ is similar to $\sigma(L)$. The function $\sigma(N)$ exhibits a clear sawlike structure of the usual QSE at not very high $y$ for all correlators. With increasing $y$, the sawteeth disappear first for the Gaussian correlator $G$ and then for the power-law correlators $\mu_{i}$, but persist for the powerlaw correlators in momentum space $\lambda_{i}$. Instead, at large $y$ the functions $f_{N}(z, y=$ const $)$ for Gaussian and power-law inhomogeneities exhibit a new type of QSE oscillations similar to that for $f_{L}(z, x=$ const $)$ in Sec. IV B. The positions of these oscillations can be found from Eqs. (38) and (39) after the substitution $x=y z$.

This effect is illustrated in Fig. 10 (the labeling of the curves is explained at the end of Sec. IV A). The figure pre-

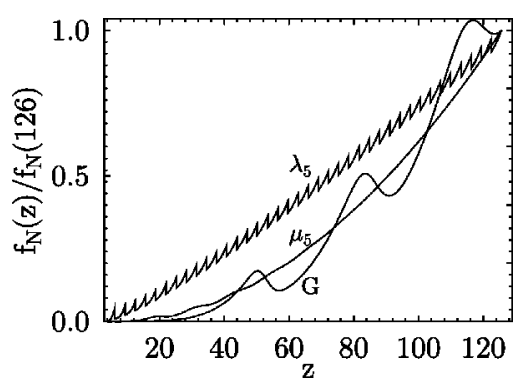

FIG. 10. Normalized function $f_{N}(z, y=20)$, Eq. (34), $f_{N}(z) / f_{N}(z=126)$, for three surface correlators. The normalization coefficients are curve $G, f_{L}(126)=1.1 \times 10^{9}$; curve $\mu_{5}, f_{L}(126)$ $=4.5 \times 10^{7}$; curve $\lambda_{5}, f_{L}(126)=1.4 \times 10^{4}$. sents functions $f_{N}(z, y=20)$, Eq. (34), for the Gaussian (curve $G$ ) and power-law $\left(\mu=0.5\right.$, curve $\left.\mu_{5}\right)$ correlators and for the correlator with a power-law power spectrum $(\lambda$ $=0.5$, curve $\lambda_{5}$ ). To compensate for different orders of magnitude of the data for these correlators, the functions are normalized by their values at $z=126, f_{N}(z) / f_{N}(z=126)$. Curve $\lambda_{5}$ exhibits a sawlike behavior typical to the usual QSE with period $\pi$ along the $z$ axis. Curves $G$ and $\mu_{5}$ exhibit new QSE oscillations with a much larger period.

\section{E. Interwall correlation of inhomogeneities and quantum size effect}

Surprisingly, the possibility of interwall correlation of surface inhomogeneities gives an interesting insight into the usual and new QSE's and provides an additional proof for our explanation of QSE oscillations reported above. The study of the effect of interwall correlation of inhomogeneities has been initiated in Ref. 12 for Gaussian correlations. Below we supplement those results for other types of surface correlators with an emphasis on the new QSE.

To decrease the number of parameters, we assume that, as in Ref. 12, the correlation functions of inhomogeneities on both walls $\zeta_{11}$ and $\zeta_{22}$ are given by the same function $\zeta_{11}(s)=\zeta_{22}(s)=\zeta(s)$. The structure of the interwall correlator of inhomogeneities, $\zeta_{12}(s)$, is assumed to be the same as for the intrawall correlations with the same correlation radius $R$. However, the amplitude $a$ of the interwall correlations is different from the intrawall ones:

$$
\zeta_{11}=\zeta_{22}=\zeta(s), \quad \zeta_{12}(s)=a \zeta(s)
$$

To compare the effect of such interwall correlations for different classes of the function $\zeta(s)$, we calculate the relative change of conductivity $\sigma$ (i.e., functions $f_{L}, f_{R}, f_{N}$ ) caused by the introduction of such correlations:

$$
\phi^{(a)}=\frac{f^{(a)}-f}{f},
$$

where $f^{(a)}$ and $f$ are the functions $f_{L, R, N}$ calculated with and without interwall correlations. An additional benefit is that the functions $\phi^{(a)}$ for all types of correlators are automatically normalized thus eliminating a difference by orders of magnitude between the functions $f_{L, R, N}$ for different types of correlation functions.

In the presence of such interwall correlations, the transition probabilities $W_{j j^{\prime}}\left(\mathbf{q}, \mathbf{q}^{\prime}\right)$, Eq. (17), become proportional, in accordance with Ref. 12 , to

$$
2\left[1+a(-1)^{j+j^{\prime}}\right] \zeta\left(\left|\mathbf{q}_{j}-\mathbf{q}_{j^{\prime}}^{\prime}\right|\right) .
$$

The most interesting effects of the interwall correlations are related to the oscillating structure of the term with $a$ in Eq. (42). If the interband transition probabilities $W_{j \neq j^{\prime}}\left(\mathbf{q}, \mathbf{q}^{\prime}\right)$ are large i.e., if $\zeta\left(\left|\mathbf{q}_{j}-\mathbf{q}_{j^{\prime}}^{\prime}\right|\right)$ is not small for $j^{\prime} \neq j$, then the contribution of the term with $a$ in Eq. (42) has a different sign for different $W_{j j^{\prime}}$ depending on whether $j+j^{\prime}$ is even or 


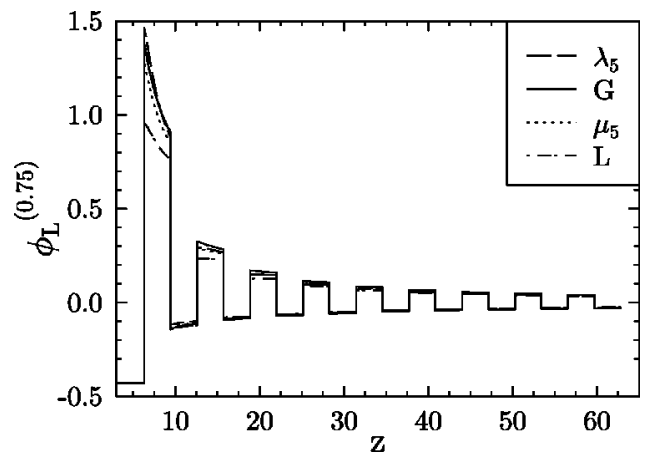

FIG. 11. Relative change $\phi_{L}^{(0.75)}$, Eq. (41), of the function $f_{L}(z, x=1)$, Eq. (32), for the interwall correlation amplitude (40), $a=0.75$, for various correlation functions of surface inhomogeneities. The labeling of the curves is explained at the end of Sec. IV A. All curves exhibit almost identical oscillations as it should be for a well-developed usual QSE.

odd. This should result in an oscillating structure of the function $\phi^{(a)}$, Eq. (41), as a function of the number of occupied minibands $S$, i.e., as a function of film thickness $L$ (the existence of such oscillations was first reported in Ref. 12 for Gaussian inhomogeneities). The period of such oscillations should be equal to that for the standard QSE and their amplitude should decrease rapidly with increasing $L$. Since our explanation of the standard QSE ties it to large interband transitions, the oscillation nature of the function $\phi^{(a)}$, Eq. (41), should exist in the same range of parameters as the standard QSE. In accordance with Sec. IV B, these oscillations should be noticeable for the function $\phi_{L}^{(a)}(z, x$ $=$ const) at small $x$ for all types of surface correlators. This is illustrated in Fig. $11(x=1)$ for the correlators $G, L, \lambda_{5}, \mu_{5}$. The figure is plotted for $a=0.75$. The similarity of the functions $\phi_{L}^{(0.75)}(z, x=1)$ is striking, but not surprising. The flat part of all curves at small $z$ is explained below. At higher values of $x$, the interband transitions [off-diagonal $W_{j j^{\prime}}$, Eq. (42)] become more and more suppressed. When the interband transitions become negligible, the only nonzero scattering probabilities are diagonal $W_{j j}$ that are proportional to $2[1+a] \zeta\left(\left|\mathbf{q}_{j}-\mathbf{q}_{j}^{\prime}\right|\right)$, Eq. (42). Since all $W_{j j}$ are scaled by the same factor $1+a$ and the conductivity is inversely proportional to $W$, the function $\phi_{L}^{(a)}(z)$ in the absence of the interband transition becomes a constant:

$$
\phi_{L}^{(a)}(z)=\frac{1}{1+a}-1
$$

If $a=0.75$, the value of this constant is $\phi_{L}^{(0.75)}(z)=-3 / 7$. Equation (43) also describes the initial part of all curves $\phi_{L}^{(a)}(z)$ for all values of $x$ at small $z$ when only the first miniband is occupied, $S=j=j^{\prime}=1$. This explains all curves in Fig. 11 having identical flat parts at small $z$.

Figure 12 illustrates $\phi_{L}^{(a)}(z, x=$ const $)$ at $x=400$ and $a$ $=0.75$ for several correlators. At this value of $x$, the exponential correlator $\lambda_{5}$, Eq. (7), exhibits, according to the results and explanation of Sec. IV B, the usual QSE. Therefore, the function $\phi_{L}^{(0.75)}(z, x=400)$ for this correlator should have

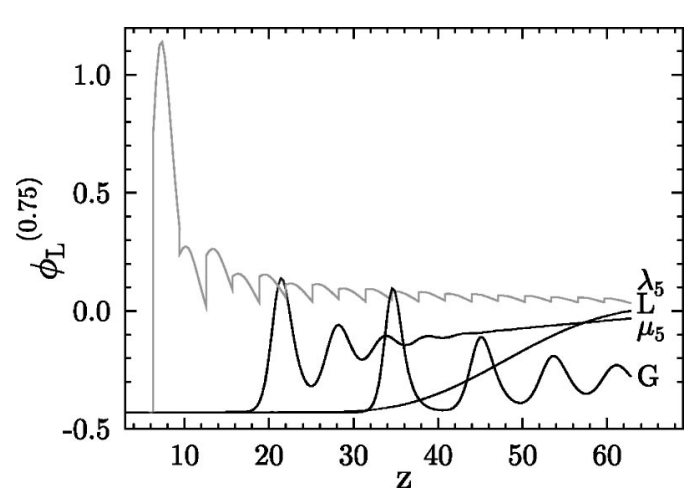

FIG. 12. Relative change $\phi_{L}^{(0.75)}$, Eq. (41), of the function $f_{L}(z, x=400)$, Eq. (32), for the interwall correlation amplitude (40) $a=0.75$ for four correlation functions of surface inhomogeneities. The labeling of the curves is explained at the end of Sec. IV A. Curve $\lambda_{5}$ exhibits oscillations in accordance with the usual QSE for curve $\lambda_{5}$ in Fig. 6. Curves $G, L$, and $\mu_{5}$ are flat at small $z, \phi_{L}^{(0.75)}$ $=-3 / 7$, Eq. (43). Oscillations on curves $G$ and $\mu_{5}$ confirm the explanation of the new QSE as an exponential appearance of transitions $j \leftrightarrow j+1$ at certain values of $z$.

an oscillation structure; this is clearly seen in Fig. 12. The Gaussian and power-law correlators $G$ and $\mu_{5}$, according to Sec. IV B, ensure the absence of interband transitions at small and moderate $z$ where the function $\phi_{L}^{(0.75)}=-3 / 7$ in Fig. 12. Our explanation for the new type of QSE in Sec. IV B is an abrupt sequential appearance of noticeable interband transitions $W_{12}, W_{23}, W_{34}$, etc., at certain values of $z=z_{j}$. Since the term with $a$ in Eq. (42) is negative for all transitions $j^{\prime}=j \pm 1$, one should observe spikes in conductivity and, therefore, in the function $\phi_{L}^{(a)}$, at $z=z_{j}$. In some sense, Fig. 12 provides the best illustration for our explanation of the new QSE.

Figure 12 also provides insight into the anomalous behavior of the conductivity for Lorentzian correlation of inhomogeneities (10), curve $L$. At $z<30$, the interband transitions are suppressed and $\phi_{L}^{(0.75)}=-3 / 7$. At higher $z$, the interband transitions become more noticeable and start increasing, but very slowly. Why the curve remains smooth when a sufficient number of transitions is already visible is still a puzzle. A possible explanation is that oscillations should appear only at very large $S$ (or $z$ ) when their amplitude should be vanishingly small.

\section{SUMMARY AND CONCLUSIONS}

In summary, we compared the behavior of conductivity for various types of surface correlators in a wide range of parameters. The following conclusions can be important when analyzing the experimental data or discussing theoretical predictions.

(i) The rough shapes of the curves of the transport coefficients are similar at small and moderate $R$ for all types of correlators though the orders of magnitude of the transport coefficients and more fine details of the curves can be differ- 
ent. To make any definite conclusions from the rough shapes of the experimental curves, one should have at least some idea of the type of the correlation function of surface inhomogeneities and/or the value of the correlation radius $R$ and the amplitude of inhomogeneities $l$. Since $l$ plays the role of a scaling parameter, getting the values of parameters of surface inhomogeneities from experimental data on transport without any additional information on the correlation of inhomogeneities could result in mistakes by orders of magnitude. In the same way, the use of the wrong correlator in theoretical calculations could result in absolutely wrong predictions without evoking any warning signals from comparison of the rough shapes of experimental and theoretical curves.

(ii) The most universal feature is the shape of the curves and order of magnitude of $\sigma(R)$ near the minimum at $R / \Lambda_{F} \sim 1$. This minimum allows experimental evaluation of the correlation length of surface inhomogeneities $R$ without any assumptions about the type of the correlation function.

(iii) The shape of the curves $\sigma(L), \sigma(N)$, and $\sigma(R)$ becomes very sensitive to the type of surface correlator at a large correlation radius of inhomogeneities, $R$. Experimentally, this is important for better quality films (see, for example, in Ref. 22) in which STM and other usual methods are not well suited for the study of the long-range behavior of the thickness fluctuations. Here transport measurements can be used as a good alternative for identification and analysis of the thickness fluctuations.

(iv) The underlying reason is very high sensitivity of coupling between quantum well states with low quantum numbers to film thickness and the long-range behavior of the thickness fluctuations. This phenomenon is quite general and should lead to observable effects not only in metal films, but for other types of quantum wells such as semiconductor films or quantum wave guides. ${ }^{23}$

(v) The persistence of the sawlike dependence of the transport coefficients on the thickness of the film, Fermi momentum, or density of fermions should signal the long-range nature of the surface correlations in momentum space $\zeta(q)$. The observation of the sawlike structure for $R>L$ is a distinct signature of the power-law decay of the power spectral density function $\zeta(q)$, though, by itself, is insufficient to make conclusions about the index in this power law. The easy suppression of the sawlike behavior points at the exponential decay of the power spectral density. The rate of this suppression is significantly different for simple exponential and Gaussian decays of $\zeta(q)$.

(vi) Thickness fluctuations with Gaussian correlations and correlations with exponential power spectrum lead to a new type of QSE in $\sigma(L), \sigma(N)$, and $\sigma(R)$ for surface inhomogeneities of a relatively large size $R$. This new QSE produces large oscillations in $\sigma(L)$ and $\sigma(N)$ and steps in dependence $\sigma(R)$. The spacing between these new QSE anomalies provides important direct information on the correlation parameters of inhomogeneities. The peaks are almost equidistant if plotted against $z^{2}$.

(vii) In contrast to the usual sawlike QSE, the new QSE oscillations are not related directly to the quantization of momentum and are a consequence of the exponential open- ing of interband transitions between minibands with small quantum numbers at certain values of the film thickness. In some sense, the effect is reminiscent of magnetic breakthrough that describes the opening of transitions between disconnected parts of the Fermi surface.

(viii) Large period of new QSE oscillations opens the way to direct observation of the QSE in the conductivity of quantized metal films and may be responsible for experimental data in the second reference of Ref. 5. An additional experimental signature should be the appearance of these new QSE oscillations only at relatively large values of the thickness of quantized metal films.

(ix) The Gaussian correlation of inhomogeneities affects particle transport in a unique way. First, the values of the transport coefficient are, except for the smallest correlation radii, larger than for other, slower correlators by orders of magnitude. This is explained by this correlator having the shortest tails resulting in the least effective scattering. Second, this type of correlation does not exhibit a sawlike dependence of the transport coefficients on the system parameters except for small correlation radii $R$. Third, this type of correlation of the surface inhomogeneities leads to the above-mentioned new type of large-scale oscillations of the transport coefficients. The combination of these features can make the Gaussian correlator readily identifiable in transport experiments.

(x) The Lorentzian correlation of inhomogeneities in configuration space is also readily identifiable by several abnormal features. The combination of these features could be another manifestation of an "unphysical" nature of this correlator. If possible, this correlator should be avoided in theoretical and computational models. A power-law correlator (8) with small index $\mu$ can serve as a good replacement in the calculations.

(xi) The results explain the observed difference in powerlaw regimes of the thickness dependence of the conductivity $\sigma(L)$ between ultrathin and more thicker films.

(xii) The relative contribution of the interwall correlation of surface inhomogeneities strongly depends on the type of QSE. For the usual QSE, the contribution of the interwall correlations is a rapidly decaying oscillation function of the film thickness. For a QSE of the new type, this contribution is constant in a wide range of small and moderate thicknesses, and becomes an oscillating function with a big period in a limited range of large thicknesses.

\section{ACKNOWLEDGMENT}

The work is supported by NSF Grant No. DMR-0077266.

\section{APPENDIX A: TRANSITION PROBABILITIES}

Various correlation functions from Sec. II allow different degrees of analytical calculations of the scattering probabilities. The angular harmonics of the correlation function $\zeta\left(\left|\mathbf{q}-\mathbf{q}^{\prime}\right|\right)$ in the transport equation (19) are defined as

$$
\zeta\left(\left|\mathbf{q}-\mathbf{q}^{\prime}\right|\right)=\frac{1}{2} \zeta^{(0)}\left(q, q^{\prime}\right)+\sum_{s=1}^{\infty} \zeta^{(s)}\left(q, q^{\prime}\right) \cos (s \chi),
$$




$$
\zeta^{(s)}=\frac{1}{\pi} \int_{0}^{2 \pi} \zeta(\chi) \cos (s \chi) d \chi
$$

where $\chi$ is the angle between the $2 \mathrm{D}$ vectors $\mathbf{q}$ and $\mathbf{q}^{\prime}$.

The harmonics for the Gaussian correlator (5) are

$$
\begin{aligned}
& \zeta^{(0)}\left(q_{j}, q_{j^{\prime}}\right)=4 \pi l^{2} R^{2}\left[e^{-Q Q^{\prime}} I_{0}\left(Q Q^{\prime}\right)\right] e^{-\left(Q-Q^{\prime}\right)^{2} / 2}, \\
& \zeta^{(1)}\left(q_{j}, q_{j^{\prime}}\right)=4 \pi l^{2} R^{2}\left[e^{-Q Q^{\prime}} I_{1}\left(Q Q^{\prime}\right)\right] e^{-\left(Q-Q^{\prime}\right)^{2} / 2},
\end{aligned}
$$

where $Q=q_{j} R, Q^{\prime}=q_{j^{\prime}} R$. Note, that in Refs. $12-14$ we used equivalent expressions with hypergeometric functions instead of modified Bessel functions. Expressions in square brackets in Eqs. (A1) are smooth functions of $Q$ and $Q^{\prime}$. The exponential coefficients $\exp \left[-\left(Q-Q^{\prime}\right)^{2} / 2\right]$, on the other hand, are rapidly going to zero for large $q R$ if $q_{j} \neq q_{j^{\prime}}$. This explains why the off-diagonal scattering probabilities $W_{j j^{\prime}}$ are much smaller than the diagonal ones at large $q R$. Such a drastic difference between interband and intraband scattering probabilities is a unique feature of the Gaussian correlator. The physical consequences are discussed in Sec. IV.

For the exponential correlator (7) the harmonics are

$$
\begin{gathered}
\zeta^{(0)}\left(q_{j}, q_{j^{\prime}}\right)=\frac{8 l^{2} R^{2} E(\Omega)}{\left[1+\left(Q-Q^{\prime}\right)^{2}\right] \sqrt{1+\left(Q+Q^{\prime}\right)^{2}}}, \\
\zeta^{(1)}\left(q_{j}, q_{j^{\prime}}\right) \\
=\frac{4 l^{2} R^{2}}{Q Q^{\prime}} \frac{\left(1+Q^{2}+Q^{\prime 2}\right) E(\Omega)-\left[1+\left(Q-Q^{\prime}\right)^{2}\right] K(\Omega)}{\left[1+\left(Q-Q^{\prime}\right)^{2}\right] \sqrt{1+\left(Q+Q^{\prime}\right)^{2}}}, \\
\Omega=2 \sqrt{Q Q^{\prime} /\left[1+\left(Q+Q^{\prime}\right)^{2}\right]},
\end{gathered}
$$

where $E$ and $K$ are complete elliptic integrals. Here the diagonal and off-diagonal transition probabilities (probabilities of the intraband and interband scattering) differ mainly by the terms $1+\left(Q-Q^{\prime}\right)^{2}$ in the denominator that are insignificant in comparison with the exponential factors for the Gaussian correlator above. The physical consequences are discussed in Sec. IV.

The power-law (8) correlation functions correspond to

$$
\begin{gathered}
\zeta^{(0)}=4 l^{2} R^{2} \sum_{m=0}^{\infty}(\mu+m) \frac{K_{\mu+m}\left(Q_{\max }\right)}{Q_{\max }^{\mu}} \frac{I_{\mu+m}\left(Q_{\min }\right)}{Q_{\min }^{\mu}} \\
\times \int_{0}^{2 \pi} C_{m}^{\mu}(\cos \phi)\left[Q^{2}+Q^{\prime 2}-2 Q Q^{\prime} \cos \phi\right]^{\mu} d \phi \\
\zeta^{(1)}=4 l^{2} R^{2} \sum_{m=0}^{\infty}(\mu+m) \frac{K_{\mu+m}\left(Q_{\max }\right)}{Q_{\max }^{\mu}} \\
\quad \times \frac{I_{\mu+m}\left(Q_{\min }\right)}{Q_{\min }^{\mu}} \int_{0}^{2 \pi} C_{m}^{\mu}(\cos \phi) \\
\quad \times\left[Q^{2}+Q^{\prime 2}-2 Q Q^{\prime} \cos \phi\right]^{\mu} \cos \phi d \phi
\end{gathered}
$$

where $C_{m}^{\mu}$ are the ultraspherical (Gegenbauer) polynomials, and $Q_{\max }=\max \left(Q, Q^{\prime}\right)$ and $Q_{\min }=\min \left(Q, Q^{\prime}\right)$. The offdiagonal transition probabilities disappear exponentially at large $\left|Q-Q^{\prime}\right|$, approximately as $\left(\left|Q-Q^{\prime}\right|\right)^{\mu-1 / 2} \exp (-\mid Q$ $\left.-Q^{\prime} \mid\right)$, i.e., much slower than for the Gaussian correlator (A2) but faster than for the correlator (A3).

The integrals in Eqs. (8) can be simplified for the Lorentzian correlator:

$$
\begin{aligned}
& \zeta^{(0)}\left(Q, Q^{\prime}\right)=8 \pi l^{2} R K_{0}\left(Q_{\max }\right) I_{0}\left(Q_{\min }\right), \\
& \zeta^{(1)}\left(Q, Q^{\prime}\right)=4 \pi l^{2} R K_{1}\left(Q_{\max }\right) I_{1}\left(Q_{\min }\right) .
\end{aligned}
$$

Note that the function $K_{0}(Q)$ diverges logarithmically at $Q$ $\rightarrow 0$. This divergence is discussed in Secs. II and IV.

The expressions for the harmonics (A4) can also be simplified for the Staras correlator, $\mu=1$, when $C_{n}^{1}(\cos \phi)$ $=\sin [(n+1) \phi] / \sin \phi$,

$$
\begin{gathered}
\int_{0}^{2 \pi} C_{m}^{1}(\cos \phi) d \phi=[0, \quad m=2 k+1 ; \quad 2 \pi, \quad m=2 k] \\
\int_{0}^{2 \pi} C_{m}^{1}(\cos \phi) \cos \phi d \phi=[0, \quad m=2 k ; \quad 2 \pi, \quad m=2 k \\
+1],
\end{gathered}
$$

and the harmonics (A4) reduce to the rapidly converging sums of the Bessel functions with alternating coefficients. For all other power-law correlators with different values of $\mu$ the integration should be performed numerically.

The last group of correlators involves power-law behavior in momentum space, Eq. (11). This group includes the Lorentzian in momentum space $\lambda=0$ that was observed in Ref. 2 and the exponential correlator (7), (A3) at $\lambda=1 / 2$. In general, the angular harmonics are

$$
\begin{aligned}
& \zeta^{(0)}=\frac{4 \pi l^{2} R^{2}}{\left[1+\left(Q^{2}-Q^{\prime 2}\right)^{2}+2\left(Q^{2}+Q^{\prime 2}\right)\right]^{(1+\lambda) / 2}} P_{\lambda}(\Omega), \\
& \zeta^{(1)}=\frac{4 \pi l^{2} R^{2} / \lambda}{\left[1+\left(Q^{2}-Q^{\prime 2}\right)^{2}+2\left(Q^{2}+Q^{\prime 2}\right)\right]^{(1+\lambda) / 2}} P_{\lambda}^{1}(\Omega), \\
& \Omega=\left(1+Q^{2}+Q^{\prime 2}\right) / \sqrt{1+\left(Q^{2}-Q^{\prime 2}\right)^{2}+2\left(Q^{2}+Q^{\prime 2}\right)}
\end{aligned}
$$

where $P_{\lambda}^{n}(\Omega)$ are the associated Legendre functions of the first kind. Note that the argument $\Omega$ of the Legendre functions in our expressions is larger than 1. One should be cautious when doing calculations with expressions (A6): some of the handbooks (and software packages, e.g., MATHEMATICA) do not use the same normalization for Legendre polynomials and Legendre functions, i.e., for functions $P_{\lambda}^{n}(\Omega)$ with integer and noninteger $\lambda$.

In the case of the Lorentzian in momentum space, $\lambda=0$,

$$
\zeta_{i k}(s)=l^{2} K_{0}(s / R), \quad \zeta(Q)=\frac{2 \pi l^{2} R^{2}}{1+(Q R)^{2}}
$$


the harmonics

$$
\begin{aligned}
& \zeta^{(0)}\left(q_{j}, q_{j^{\prime}}\right)=\frac{4 \pi l^{2} R^{2}}{\sqrt{1+\left(Q^{2}-Q^{\prime 2}\right)^{2}+2\left(Q^{2}+Q^{\prime 2}\right)}}, \\
& \zeta^{(1)}\left(q_{j}, q_{j^{\prime}}\right) \\
& =\frac{8 \pi l^{2} R^{2} Q Q^{\prime}}{\sqrt{1+\left(Q^{2}-Q^{\prime 2}\right)^{2}+2\left(Q^{2}+Q^{\prime 2}\right)}} \\
& \quad \times \frac{1}{1+Q^{2}+Q^{\prime 2}+\sqrt{1+\left(Q^{2}-Q^{\prime 2}\right)^{2}+2\left(Q^{2}+Q^{\prime 2}\right)}} .
\end{aligned}
$$

Note that this correlator diverges in real space at $s \rightarrow 0$.

\section{APPENDIX B: POSITIONS OF NEW QSE OSCILLATIONS}

The peak positions are determined by the condition that the absolute value of the diagonal and the first off-diagonal matrix elements in transport equation (19) become comparable:

$$
1 / \tau_{j, j+1} \sim 1 / \tau_{j j}
$$

Rewriting this condition via transition probabilities $W_{j j^{\prime}}^{(0,1)}\left(\mathbf{q}, \mathbf{q}^{\prime}\right)$ we get

$$
\left[W_{j j}^{(0)}(x, z)-W_{j j}^{(1)}(x, z)\right]+\sum_{j^{\prime} \neq j} W_{j, j^{\prime}}^{(0)}(x, z) \sim W_{j, j+1}^{(1)}(x, z)
$$

where $W_{j j^{\prime}}^{(0,1)}\left(q_{j}, q_{j^{\prime}}\right)$ are the zeroth and first harmonics of $W\left(\mathbf{q}_{j}-\mathbf{q}_{j^{\prime}}\right)$ over the angle $\widehat{\mathbf{q}}_{j} \mathbf{q}_{j^{\prime}}$ that can be expressed explicitly via the surface correlation functions [see Eq. (17) and Appendix A]. For large $q_{j} R$, the off-diagonal scattering probabilities $W_{j j^{\prime}}$ are exponentially suppressed for Gaussian and power-law inhomogeneities, Eqs. (A2) and (A4): $W_{j j}^{(0)}$ $\sim W_{j j}^{(1)} \gg W_{j, j+1}^{(0)} \sim W_{j, j+1}^{(1)}$. With logarithmic accuracy, the condition (B1) corresponds to the equation

$$
W_{j j}^{(0)}(x, z)-W_{j j}^{(1)}(x, z)=W_{j, j+1}^{(0)}(x, z) .
$$

Taking into consideration the asymptotic behavior for modified Bessel functions in Eq. (A2) for the Gaussian correlator, Eq. (B2) can be reduced to

$$
\frac{j^{2}}{2 Q_{j}^{3}}=\frac{(j+1)^{2}}{\sqrt{Q_{j} Q_{j+1}}} \exp \left[-\frac{1}{2}\left(Q_{j}-Q_{j+1}\right)^{2}\right]
$$

where $Q_{j}=x \sqrt{1-(\pi j / z)^{2}}$. When $z / \pi j \gg 1$, we can put $Q_{j}$ $\approx Q_{j+1} \approx x$ in the denominator. The exponent should be evaluated more carefully: $Q_{j}-Q_{j+1} \approx x \pi^{2}(2 j+1) / 2 z^{2}$. Then Eq. (B3) yields the following values of the peak positions:

$$
z_{j}(x)=\frac{\pi}{2} \frac{\sqrt{x(2 j+1)}}{\left[\ln \left(x \sqrt{2} \frac{1+j}{j}\right)\right]^{1 / 4}} .
$$

Since $x=y z$, these peak positions $z_{j}(x)$ can also be used to get the peak positions for the conductivity at fixed $y, z_{j}(y)$ as a solution of the following algebraic equation:

$$
z_{j}(y)=\frac{\pi^{2}}{4} \frac{y(2 j+1)}{\left[\ln \left(z_{j}(y) y \sqrt{2} \frac{1+j}{j}\right)\right]^{1 / 2}} .
$$

Similar but more cumbersome calculations, can be performed for the power-law correlators (8). For example, if $\mu=1 / 2$, Eq. (B2) reads

$$
\begin{aligned}
& 4 j^{2} \int_{0}^{\pi / 2} \exp \left(-2 Q_{j} \sin t\right) \sin ^{2} t d t \\
& \quad=2(j+1)^{2} \int_{0}^{\pi / 2} \exp \left(-\sqrt{\nu^{2}+4 Q_{j} Q_{j+1} \sin ^{2} t}\right) d t,
\end{aligned}
$$

where we introduced $\nu \equiv \nu_{j, j+1}=Q_{j}-Q_{j+1}$. For large $Q_{j}$, an asymptotic estimate for the integral in the left-hand side is $1 / 4 Q_{j}^{3}$. A rough asymptotic estimate for the integral in the right-hand side of the equation is

$$
\begin{aligned}
& \int_{0}^{1} \exp \left(-\sqrt{\nu^{2}+4 Q_{j} Q_{j+1} t^{2}}\right) \frac{d t}{\sqrt{1-t^{2}}} \\
& \quad \approx \frac{1}{2 \sqrt{Q_{j} Q_{j+1}}} \int_{0}^{2 \sqrt{Q_{j} Q_{j+1}}} \exp \left(-\sqrt{\nu^{2}+y^{2}}\right) d y .
\end{aligned}
$$

In order to estimate this integral, we can substitute $\sqrt{\nu^{2}+y^{2}}$ by

$$
\sqrt{\nu^{2}+y^{2}} \rightarrow \begin{cases}\nu, & \text { for } y<\nu \\ y, & \text { for } y>\nu\end{cases}
$$

Then

$$
\frac{1}{2 Q_{j}} \int_{0}^{\infty} \exp \left(-\sqrt{\nu^{2}+y^{2}}\right) d y \approx \frac{1}{2 Q_{j}} e^{-\nu}(\nu+1) .
$$

This leads to the following estimate for the peak positions:

$$
z_{j}(x)=\pi \sqrt{\frac{x(2 j+1)}{2 \nu_{j}}}
$$

where $\nu_{j}$ is the root of the transcendental equation

$$
\nu_{j}=2 \ln A_{j}+\ln \left(1+\nu_{j}\right), \quad A_{j} \equiv x(1+1 / j) .
$$

The last equation can be solved by iterations: 


$$
\begin{gathered}
\nu=\nu^{(0)}+\nu^{(1)}+\nu^{(2)}+\cdots, \\
\nu^{(0)}=2 \ln A, \quad \nu^{(1)}=\ln [2 \ln A+1], \ldots .
\end{gathered}
$$$$
z_{j}=\frac{\pi}{2} \sqrt{\frac{x(2 j+1)}{\ln \left[A_{j}\left(2 \ln A_{j}\right)^{\mu / 2+1 / 4}\right]}}
$$

Finally, with logarithmic accuracy, the solution of Eq. (B6) for the positions of peaks becomes

$$
z_{j}=\frac{\pi}{2} \sqrt{\frac{x(2 j+1)}{\ln \{x \sqrt{\ln [x(1+1 / j)]}(1+1 / j)\}}} .
$$

Similar asymptotic estimates for the power-law correlators with arbitrary $\mu$ yield

$$
A_{j}=x \frac{1+j}{j} \sqrt{\frac{2}{\Gamma(\mu+5 / 2)}} .
$$

It is clear from Eq. (B9) that the dependence of the peak positions on $\mu$ is extremely weak.
${ }^{1}$ J. A. Ogilvy, Theory of Wave Scattering from Random Surfaces (Adam Hilger, Bristol, 1991).

${ }^{2}$ R. M. Feenstra et al., Phys. Rev. Lett. 72, 2749 (1994).

${ }^{3}$ G. Fishman and D. Calecki, Phys. Rev. B 43, 11581 (1991).

${ }^{4}$ G. Palasantzas and J. Barnas, Phys. Rev. B 56, 7726 (1997); G. Palasantzas, Y.-P. Zhao, G.-C. Wang, T.-M. Lu, J. Barnas, and J. Th. M. De Hosson, ibid. 61, 11109 (2000).

${ }^{5}$ M. Jałochowski, M. Hoffmann, and E. Bauer, Phys. Rev. Lett. 76, 4227 (1996); Phys. Rev. B 51, 7231 (1995); L. A. Kuzik, Yu. E. Petrov, F. A. Pudonin, and V. A. Yakovlev, Sov. Phys. JETP 78, 114 (1994); G. M. Mikhailov, I. V. Malikov, and A. V. Chernykh, JETP Lett. 66, 725 (1997).

${ }^{6}$ J. J. Paggel, T. Miller, and T. C. Chang, Science 283, 1709 (1999); D. A. Evans, M. Alonso, R. Cimino, and K. Horn, Phys. Rev. Lett. 70, 3483 (1993); J. E. Ortega, F. J. Himpsel, G. J. Mankey, and R. F. Willis, Phys. Rev. B 47, 1540 (1993).

${ }^{7}$ S. Andrieu, C. Chatelain, M. Lemine, B. Berche, and Ph. Bauer, Phys. Rev. Lett. 86, 3883 (2001).

${ }^{8}$ I. B. Altfeder, D. M. Chen, and K. A. Matveev, Phys. Rev. Lett. 80, 4895 (1998); 78, 2815 (1997).

${ }^{9}$ V. B. Sandomirskii, Zh. Éksp. Teor. Fiz. 52, 158 (1968) [Sov. Phys. JETP 25, 101 (1967)].

${ }^{10}$ N. Trivedi and N.W. Ashcroft, Phys. Rev. B 38, 12298 (1988).

${ }^{11}$ M. Jałochowski, E. Bauer, H. Knoppe, and G. Lilienkamp, Phys. Rev. B 45, 13607 (1992); M. Jałochowski, M. Hoffmann, and E. Bauer, ibid. 51, 7231 (1995); H. Sakaki, T. Noda, K. Hirakawa, M. Tanaka, and T. Matsusue, Appl. Phys. Lett. 51,
1934 (1987); L.-W. Tu, G. K. Wong, and J. B. Ketterson, ibid. 55, 1327 (1989).

${ }^{12}$ A. E. Meyerovich and A. Stepaniants, Phys. Rev. B 58, 13242 (1998).

${ }^{13}$ A. E. Meyerovich and A. Stepaniants, Phys. Rev. B 60, 9129 (1999).

${ }^{14}$ A. E. Meyerovich and A. Stepaniants, J. Phys.: Condens. Matter 12, 5575 (2000).

${ }^{15}$ Z. Tesanovic, M. V. Jaric, and S. Maekawa, Phys. Rev. Lett. 57, 2760 (1986).

${ }^{16}$ G. Fishman and D. Calecki, Phys. Rev. Lett. 62, 1302 (1989).

${ }^{17}$ A. Kawabata, J. Phys. Soc. Jpn. 62, 3988 (1993).

${ }^{18}$ A. E. Meyerovich and S. Stepaniants, Phys. Rev. Lett. 73, 316 (1994); Phys. Rev. B 51, 17116 (1995); J. Phys.: Condens. Matter 9, 4157 (1997).

${ }^{19}$ N. M. Makarov, A. V. Moroz, and V. A. Yampol'skii, Phys. Rev. B 52, 6087 (1995)

${ }^{20}$ A. E. Meyerovich and A. Stepaniants, Aust. J. Phys. 53, 53 (2000).

${ }^{21}$ J. Henz, H. von Känel, M. Ospelt, and P. Wachter, Surf. Sci. 189/190, 1055 (1987); J. Y. Duboz, P. A. Badoz, E. Rosencher, J. Henz, M. Ospelt, H. von Känel, and A. Briggs, Appl. Phys. Lett. 53, 788 (1988).

${ }^{22}$ J. J. Paggel, T. Miller, and T. C. Chiang, Phys. Rev. Lett. 81, 5632 (1998); F. Patthey and W.-D. Schneider, Phys. Rev. B 50, 17560 (1994); M. Schmid et al., Phys. Rev. Lett. 76, 2298 (1996).

${ }^{23}$ J. A. Sanchez-Gil, V. Freilikher, I. Yurkevich, and A. A. Maradudin, Phys. Rev. Lett. 80, 948 (1998). 